CERN-TH/98-196

hep-ph/9806519

June, 1998

\title{
The Two-Loop Finite-Temperature Effective Potential of the MSSM and Baryogenesis
}

\author{
Marta Losada円 \\ CERN Theory Division \\ CH-1211 Geneva 23, Switzerland
}

\begin{abstract}
We construct an effective three dimensional theory for the MSSM at high temperatures in the limit of large- $m_{A}$. We analyse the two-loop effective potential of the 3D theory for the case of a light right handed stop to determine the precise region in the $m_{h}-m_{\tilde{t}_{R}}$ plane for which the sphaleron constraint for preservation of the baryon asymmetry is satisfied. We also compare with results previously obtained using $3 \mathrm{D}$ and $4 \mathrm{D}$ calculations of the effective potential. A two-stage phase transition still persists for a small range of values of $m_{\tilde{t}_{R}}$. The allowed region requires a value of $m_{\tilde{t}_{R}} \lesssim m_{t}$ and $m_{h} \lesssim 100(110) \mathrm{GeV}$ for $m_{Q}=300 \mathrm{GeV}(1 \mathrm{TeV})$.
\end{abstract}

\footnotetext{
${ }^{1}$ On leave of absence from the Universidad Antonio Nariño, Santa Fe de Bogotá, COLOMBIA.
} 


\section{Introduction}

The analysis of the electroweak phase transition in the MSSM has been the subject of intensive study in recent years. The main motivation is the generation (and preservation) of a possible baryon asymmetry at the electroweak scale [1, 2] (for reviews, see [3]-[5]). Many different contributions have given a clearer idea as to where in parameter space the condition of a strong enough first-order phase transition can occur in order for electroweak baryogenesis to be possible. Mainly two different analytic approaches have been used in the analysis of the phase transition for the MSSM. The first one involves the evaluation of the effective potential in the 4D theory. The one-loop analysis [6]- [9] led to the conclusion that low values of the ratio of the vacuum expectation values of the two Higgs doublets $\tan \beta=\frac{v_{2}}{v_{1}}$, and large values of the pseudoscalar mass $m_{A}$ were favoured. More recently the effects of a very light right handed stop with a soft SUSY-breaking mass value of $m_{U}^{2} \lesssim 0$ were shown to strongly strengthen the phase transition by enhancing the cubic term in the effective potential [10, 11]. As first pointed out by Espinosa [12], two-loop corrections were also shown to be very important in order to increment the allowed parameter space for which electroweak baryogenesis can take place. In particular two-loop QCD corrections from stops can strongly affect the value of the scalar field at the phase transition [12]-[14]. A precise determination of the region in parameter space for which electroweak baryogenesis is viable was done by Carena et al. [15, using a twoloop calculation in 4D, with a light right handed stop, and a heavy third generation left squark doublet, which is decoupled from the thermal bath. They conclude that the Higgs mass must be lower than $105 \mathrm{GeV}$ and that the right stop mass must be in the range of 110-160 GeV if absolute stability of the physical vacuum is required. A very recent paper by Cline and Moore [16] use the two-loop effective potential in 4D, fully incorporating squark and Higgs boson mixing, to determine the allowed region in parameter space. Their results are in good agreement with those of ref. [15.

In the second approach for the analysis of the phase transition, the aim is to separate the perturbative and non-perturbative aspects of the theory. The purely perturbative component of the calculation is performed by constructing an effective 3D theory for the model under consideration [17]- 23]. The parameters in the 3D Lagrangian are obtained using dimensional reduction at high temperature by matching the static Green's functions in the two theories, to a given order in the perturbative expansion, by integrating out the non-zero Matsubara modes with masses of the order 
of $\pi T$, where $T$ is the temperature. A further reduction can also be performed noting that some of the static modes in the theory have acquired thermal masses proportional to a gauge coupling multiplied by the temperature, $\sim g_{w} T, g_{s} T$. These so-called heavy particles can then be integrated out as well. The effective potential calculated in the 3D theory reproduces the results obtained with the $4 \mathrm{D}$ effective potential. References [21], [24]-26] give more details concerning the construction of effective theories for both the Standard Model and the MSSM. This approach simplifies the theory as it is now purely bosonic, which facilitates lattice simulations. Numerical studies of the reduced theory will take into account the non-perturbative effects. We refer the reader to the above publications for further discussion regarding strengths and weaknesses of 4D and 3D calculations.

For the generic case in which there is a single light scalar doublet field at the phase transition the constraint on strength of the transition is translated into an upper bound on the ratio of the 3D Higgs self-coupling and the square 3D gauge coupling $\frac{\lambda_{3}}{g_{w_{3}}^{2}}$ [21. This ratio has a weak dependence on the temperature for values close to the critical temperature of the phase transition for the allowed range of values of the Higgs mass [27]. In addition, the 3D scalar and gauge couplings are renormalization group invariant. This implies that a 1-loop matching of the 3D coupling constants and masses to the physical parameters and the temperature suffices to determine the strength of the phase transition, using the constraint given by the non-perturbative analysis of the phase transition for a single light scalar field [21].

To clarify an essential point, we recall that the critical temperature for the transition from the high temperature minimum to the standard electroweak minimum is obtained from the condition that the value of the effective potential at these two minima are equal, $V(0, T)=V\left(\phi_{c}, T\right)$. Therefore we insist that the value of the critical temperature does depend on a precise determination of the $3 \mathrm{D}$ mass parameter, which is not renormalization group invariant. The scalar mass requires ultraviolet renormalization and a 2-loop calculation (in 4D) must be performed even for the case of a single light scalar at the phase transition. However, since the ratio of the vacuum expectation value of the scalar field to the temperature, $\frac{\phi_{c}}{T_{c}}$ (or equivalently, $\frac{\lambda_{3}}{g_{w_{3}}^{2}}$ ), which determines the rate of sphaleron transitions in the broken phase has only a weak dependence, in this case, on the temperature, the two-loop calculation is not necessary.

In the initial analysis for the MSSM using the 3D approach [24-27, the light stop scenario could only be investigated for values of the right handed stop soft SUSY-breaking mass of $m_{U} \gtrsim 50$ 
$\mathrm{GeV}$. For lower values of the stop mass the perturbative procedure of integrating out the "heavy" modes starts to break down as the relevant expansion parameter is of the form $\frac{g_{s_{3}}^{2}}{m_{U_{3}}}$. In addition, the numerical constraint from non-perturbative studies is no longer valid and new studies that include the effect of the $\mathrm{SU}(3)$ gauge fields must be performed.

An effective Lagrangian for a light Higgs and a light stop must be constructed to analyse the phase transition for lower values of $m_{U}$ within the 3D approach [14]. A surprising result of the perturbative analysis in this scenario was that a possible two-stage phase transition could take place, in which the Universe would first undergo a transition to a colour-breaking vacuum and, at a lower temperature, another transition to the physical vacuum would occur. In fact, the work of Bodeker et al. was the first to point out that, for a small range of values of $m_{\tilde{t}_{R}}$, the phase transition could occur in two stages for the MSSM. This analysis was based on a 2-loop calculation of the effective potential in 3D including the leading corrections in the dimensional-reduction procedure.

It is of course of great interest to exactly identify the range of parameter space for which this twostage phase transition can occur. In this case it is necessary to determine the critical temperatures very precisely for the two possible directions of the transition. The most relevant quantities that determine the critical temperatures are the 3D mass parameters for the Higgs doublet and the right handed stop. These masses depend logarithmically on two parameters, $\Lambda_{H_{3}}$ or $\Lambda_{U_{3}}$, which can only be determined exactly from the $4 \mathrm{D}$ theory. In the initial reduction implemented by [14], the exact values of $\Lambda_{H_{3}}$ and $\Lambda_{U_{3}}$ were not determined. An estimate was used instead, based on the value of the corresponding parameter in the Standard Model. Here we employ a combination of 4D and 3D calculations of the effective potential to obtain the exact values of $\Lambda_{H_{3}}$ and $\Lambda_{U_{3}}$.

Undoubtably, many of the questions that arise from the limitations of perturbation theory will only be answered when the corresponding lattice calculations are done. However, the first non-perturbative results show that the 2-loop results for the strength of the phase transition are conservative in the bounds they impose on the allowed masses for the Higgs and the light stop [14]. These results were obtained using a simplified model in the reduction procedure. For more complicated initial Lagrangians the final effective 3D theory is characterized by the same couplings and masses. We would like to point out a few of the features that can be affected in the perturbative calculation of the effective theory and their consequent effect on baryogenesis. Additionally, in order to apply the non-perturbative results, a precise mapping of the $4 \mathrm{D}$ to $3 \mathrm{D}$ parameters is needed, 
which is presented in this paper. The results obtained with the perturbative 2-loop effective potential presented here can then be compared with the lattice analysis.

The procedure of constructing an effective 3D theory is based on the mass hierarchy which appears at finite temperature. The validity of the results depends on an adequate expansion parameter and the suppression of the higher order terms in the 3D theory. The value of the masses of the particles which are integrated out will define the regime of validity of the approach. As mentioned above, previous analyses [24]-27] of the 3D theory integrating out the right handed stop claimed that for $m_{U} \gtrsim 50 \mathrm{GeV}$ the higher order terms were suppressed and consequently the effective theory was an adequate description. We compare the results obtained for the ratio of the vacuum expectation value of the Higgs field to the temperature using the two-loop effective potential with and without integrating out the right handed stop to determine more precisely the value of the right handed stop mass for which the effective theory is no longer valid. We find that for values of $m_{U} \lesssim 123 \mathrm{GeV}$ the results obtained having integrated out the right handed stop are unreliable.

The paper is organized as follows: in section 2.1 we present the dimensional reduction to the effective bosonic theory at one-loop. Section 2.2 presents a further one-loop reduction in the 3D theory, eliminating the heavy fields. In section 2.3 we give the expression for the 2-loop unresummed effective potential in $4 \mathrm{D}$, which is necessary for evaluating $\Lambda_{H_{3}}$ and $\Lambda_{U_{3}}$. The contribution to the 3D effective potential from the "heavy" particles that were integrated out at the second stage is given in section 2.4. Section 2.5 discusses the relevant zero-temperature effects that must be included in our analysis. In section 3 we present our results for the critical temperatures and the strength of the phase transition. The allowed region for electroweak baryogenesis to occur is also given here. A comparison of the results obtained from the effective potential with and without integrating out the right handed stop is given in this section. Finally, in section 4, we conclude. The appendix contains the relevant formulae for the case in which the right handed stop is integrated out.

\section{Dimensional Reduction}

We will now perform dimensional reduction by matching, as has been previously done in refs. [21], 24-26] for different models. Our initial 4D Lagrangian corresponds to the MSSM in the large$m_{A}$ limit. The particles that contribute to the thermal bath are the Standard Model particles plus 
third-generation squarks: $\tilde{t}_{L}, \tilde{b}_{L}, \tilde{t}_{R}, \tilde{b}_{R}$. We will only consider here the case of zero squark mixing. The results for non-zero squark mixing will be presented elsewhere [28]. There are two stages of reduction. The first one corresponds to the integration out of all non-zero Matsubara modes, that is with a thermal mass of the order of $\sim \pi T$. We calculate all one-loop contributions to mass terms and coupling constants of the static fields to order $g^{4}$, where $g$ denotes a gauge or top Yukawa coupling. The second stage of reduction corresponds to the integration of heavy particles with masses of the order of $g_{w} T, g_{s} T$.

\subsection{First Stage}

The potential in the 3D effective theory after integration over non-zero Matsubara modes is of the form

$$
\begin{aligned}
V & =m_{H_{3}}^{2} H^{\dagger} H+\lambda_{H_{3}}\left(H^{\dagger} H\right)^{2}+m_{U_{3}}^{2} U^{\dagger} U+\lambda_{U_{3}}\left(U^{\dagger} U\right)^{2}+\gamma_{3}\left(H^{\dagger} H\right)\left(U^{\dagger} U\right) \\
& +m_{Q_{3}}^{2} Q^{\dagger} Q+m_{D_{3}}^{2} D^{\dagger} D+\Lambda_{3}^{Q}\left(H^{\dagger} H\right)\left(Q^{\dagger} Q\right)+\Lambda_{4}^{c}\left(H^{\dagger} Q\right)\left(Q^{\dagger} H\right) \\
& +\left(\Lambda_{4}^{s}+h_{t}^{L}\right)\left|\epsilon_{i j} H^{i} Q^{j}\right|^{2}+\left(h_{t}^{Q U}+g_{s_{1}}^{Q U}\right) Q_{i \alpha}^{*} U_{\alpha}^{*} Q_{i \beta} U_{\beta} \\
& +g_{s_{2}}^{Q U} U_{\alpha} U_{\alpha}^{*} Q_{j \gamma}^{*} Q_{j \gamma}+g_{s_{1}}^{Q D} D_{\alpha} D_{\beta}^{*} Q_{j \beta}^{*} Q_{j \alpha} \\
& +g_{s_{2}}^{Q D} D_{\alpha} D_{\alpha}^{*} Q_{j \gamma}^{*} Q_{j \gamma}+g_{s_{1}}^{U D} U_{\alpha} U_{\gamma}^{*} D_{\gamma}^{*} D_{\alpha} \\
& +g_{s_{2}}^{U D} U_{\alpha} U_{\alpha}^{*} D_{\gamma}^{*} D_{\gamma}+\Lambda_{1}\left(Q^{\dagger} Q\right)^{2}+\lambda_{D_{3}}\left(D^{\dagger} D\right)^{2}+\lambda_{Q_{3}}\left(Q_{i}^{\dagger} Q_{i}\right)^{2} \\
& +g_{s_{1}}^{Q Q} Q_{i \alpha}^{*} Q_{j \alpha}^{*} Q_{i \gamma} Q_{j \gamma}+g_{s_{2}}^{Q Q} Q_{i \alpha} Q_{i \alpha}^{*} Q_{j \gamma}^{*} Q_{j \gamma} \\
& +\frac{1}{2} m_{A_{0}}^{2} A_{0}^{a} A_{0}^{a}+\frac{1}{2} m_{C_{0}}^{2} C_{0}^{A} C_{0}^{A}+\frac{1}{4} g_{w_{3}}^{2}\left(H^{\dagger} H\right)\left(A_{0}^{a} A_{0}^{a}\right) \\
& +\frac{1}{4} g_{s_{3}}^{2} C_{0}^{A} C_{0}^{B}\left(U^{*}\right)^{\dagger} \lambda^{A} \lambda^{B} U^{*}
\end{aligned}
$$

2 The effects of non-zero squark mixing in the case of a relatively light left squark doublet can complicate the calculation considerably. For a heavy left squark doublet at large values of the mixing parameters, the two-stage scenario is not realized [15. However, this effect may not persist for lower values of the mass of the left doublet as the contribution to the thermal mass of the scalars is changed, see [24, 26, 28. On the other hand, as was noticed in previous studies of the phase transition, a non-zero value of the mixing parameters always weakens the strength of the transition. So lower values of the Higgs mass or the right handed stop mass are necessary to enhance the strength of the transition for non-zero squark mixing. Thus our results give upper bounds on the scalar masses. 
Here $H$ is the Higgs doublet field, $U(D)$ is the right handed stop(sbottom) field, and $Q$ is the third generation left squark doublet field. The longitudinal components of the $\mathrm{SU}(2)$ and $\mathrm{SU}(3)$ gauge fields are denoted by $A_{o}$ and $C_{o}$, respectively. The latin (greek) indices indicate $\mathrm{SU}(2)(\mathrm{SU}(3))$ components. As usual, the fields in eq. (11) are the static components of the scalar fields properly renormalized, the dimension of the fields in $3 \mathrm{D}$ is $[\mathrm{GeV}]^{1 / 2}$. Quartic couplings are of order $g_{i}^{2}\left(h_{t}^{2}\right) T$, having dimensions of $[\mathrm{GeV}]$; here $g_{i}\left(h_{t}\right)$ denotes a gauge ( top Yukawa) coupling. In the following we have not included the correction to the quartic coupling between the doublet Higgs field and the triplet scalar field $A_{0}$, or the corresponding correction for the $\mathrm{SU}(3)$ counterparts. We work throughout in the Landau gauge. For an analysis of the gauge dependence, we refer the reader to [24].

We present the full relations between 3D coupling constants and masses in terms of the underlying 4D parameters and the temperature. Partial results for the MSSM in the large- $m_{A}$ limit can be found in ref. 14 ?.

\subsubsection{Mass terms}

For the Higgs doublet we havef

$$
\begin{aligned}
m_{H_{3}}^{2} & =m_{H}^{2}\left(1+\frac{9}{4} g_{w}^{2} \frac{L_{b}}{16 \pi^{2}}-3 h_{t}^{2} \frac{L_{f}}{16 \pi^{2}}\right) \\
& +T^{2}\left(\frac{\lambda}{2}+\frac{3}{16} g_{w}^{2}+\frac{1}{16} g^{\prime 2}+\frac{1}{4} h_{t}^{2}+\frac{1}{4}\left(2 h_{t}^{2} \sin ^{2} \beta+2 \lambda_{3}+\lambda_{4}\right)\right) \\
& -\frac{L_{b}}{16 \pi^{2}}\left(6 \lambda m_{H}^{2}+3\left(m_{Q}^{2}+m_{U}^{2}\right) h_{t}^{2} \sin ^{2} \beta\right),
\end{aligned}
$$

where the Higgs mass parameter is denoted by $m_{H}$, and $\lambda=\frac{\left(g_{w}^{2}+g^{\prime 2}\right)}{8} \cos 2 \beta, \lambda_{3}=\frac{g_{w}^{2}}{4}, \lambda_{4}=-\frac{g_{w}^{2}}{2}$, $L_{b}=2 \log \frac{\bar{\mu} e^{\gamma}}{4 \pi T} \approx 2 \log \frac{\mu}{7.055 T}, L_{f}=L_{b}+4 \log 2$. Here $\bar{\mu}$ is the mass scale defined by the modified

\footnotetext{
${ }^{3}$ If a light higgsino is included, there will be important effects that are proportional to the top Yukawa coupling in the dimensionally reduced theory [26].

${ }^{4}$ We mostly neglect the hypercharge coupling $g^{\prime}$, throughout the paper. The only exception is in the contribution to the tree-level expression of the Higgs self-coupling $\lambda$, as this latter quantity is fundamental in determining the strength of the phase transition.
} 
minimal substraction scheme $(\overline{M S})$ scheme. Similarly, for the third generation squark mass terms we have

$$
\begin{gathered}
m_{U_{3}}^{2}=m_{U}^{2}\left(1+4 g_{s}^{2} \frac{L_{b}}{16 \pi^{2}}\right)+T^{2}\left(\frac{1}{3} g_{s}^{2}+\frac{2}{3} \lambda_{U}+\frac{1}{6} h_{t}^{2} \sin ^{2} \beta+\frac{1}{6} h_{t}^{2}\right) \\
-\frac{L_{b}}{16 \pi^{2}}\left(\frac{4}{3} g_{s}^{2} m_{U}^{2}+2 h_{t}^{2} \sin ^{2} \beta\left(m_{H}^{2}+m_{Q}^{2}\right)\right) \\
m_{Q_{3}}^{2}=m_{Q}^{2}\left(1+\left(\frac{9}{4} g_{w}^{2}+4 g_{s}^{2}\right) \frac{L_{b}}{16 \pi^{2}}\right)+T^{2}\left(\frac{3}{16} g_{w}^{2}+\frac{\lambda_{1}}{2}+\frac{4}{9} g_{s}^{2}+\frac{1}{12} h_{t}^{2}\left(1+\sin ^{2} \beta\right)\right) \\
-\frac{L_{b}}{16 \pi^{2}}\left(\frac{4}{3} g_{s}^{2} m_{Q}^{2}+6 \lambda_{1} m_{Q}^{2}+h_{t}^{2} m_{U}^{2}+h_{t}^{2} \sin ^{2} \beta m_{H}^{2}\right), \\
m_{D_{3}}^{2}=m_{D}^{2}\left(1+4 g_{s}^{2} \frac{L_{b}}{16 \pi^{2}}\right)+T^{2}\left(\frac{4}{9} g_{s}^{2}\right)-\frac{L_{b}}{16 \pi^{2}}\left(\frac{4}{3} g_{s}^{2} m_{D}^{2}\right) .
\end{gathered}
$$

where the soft SUSY-breaking masses for the third generation left squark doublet and the right handed sbottom are denoted by $m_{Q}$ and $m_{D}$ respectively, and $\lambda_{U}=\frac{g_{s}^{2}}{6}, \lambda_{1}=\frac{g_{w}^{2}}{8}$. The longitudinal components of the $\mathrm{SU}(2)$ and $\mathrm{SU}(3)$ gauge fields acquire thermal masses given by

$$
\begin{aligned}
& m_{A_{0}}^{2}=g_{w}^{2} T^{2}\left(\frac{2}{3}+\frac{N_{f}}{12}+\frac{N_{s w}}{6}\right), \\
& m_{C_{0}}^{2}=g_{s}^{2} T^{2}\left(1+\frac{N_{f}}{12}+\frac{N_{s s}}{6}\right),
\end{aligned}
$$

respectively, where $N_{s w}=4, N_{s s}=4, N_{f}=6$ [14.

\subsubsection{Couplings}

The 3D gauge coupling expressions for a dimensionally reduced $S U(N)$ gauge theory can be found in ref. [14]. We include them for completeness

$$
g_{3}^{2}=T g^{2}(\bar{\mu})\left[1+\frac{g^{2}(\bar{\mu})}{48 \pi^{2}}\left(\left(22 N-N_{s}\right) \frac{L_{b}}{2}-N_{f} L_{f}+N\right)\right]
$$


Here $N_{s}$ is the number of scalar fields in the fundamental representation and $N_{f}$ is the number of fermions.

For the scalar quartic self-couplings, we have the following relations arising from the diagrams that have been shown in refs. [24, 26] ?,

$$
\begin{aligned}
\lambda_{H_{3}}= & \lambda T\left(1+\frac{9}{2} g_{w}^{2} \frac{L_{b}}{16 \pi^{2}}-6 h_{t}^{2} \sin ^{2} \beta \frac{L_{f}}{16 \pi^{2}}\right) \\
- & T\left[\frac { L _ { b } } { 1 6 \pi ^ { 2 } } \left(\frac{9}{16} g_{w}^{4}+12 \lambda^{2}+3\left(\lambda_{3}^{2}+\lambda_{3} \lambda_{4}+\lambda_{4}^{2} \cos ^{4} \beta+\lambda_{4}^{2} \sin ^{4} \beta\right.\right.\right. \\
+ & \left.\left.\left.h_{t}^{2} \sin ^{2} \beta\left(\lambda_{3}+\lambda_{4} \sin ^{2} \beta\right)+h_{t}^{4} \sin ^{4} \beta\right)\right)+\frac{3}{8} \frac{g_{w}^{4}}{16 \pi^{2}}+3 h_{t}^{4} \sin ^{4} \beta \frac{L_{f}}{16 \pi^{2}}\right], \\
\lambda_{U_{3}}= & \frac{g_{s}^{2}}{6} T\left(1+8 g_{s}^{2} \frac{L_{b}}{16 \pi^{2}}\right)-T\left[\frac { L _ { b } } { 1 6 \pi ^ { 2 } } \left(\frac{23}{36} g_{s}^{4}+\frac{13}{12} g_{s}^{4}-\frac{2}{3} h_{t}^{2} g_{s}^{4}\right.\right. \\
& \left.\left.+h_{t}^{4}+h_{t}^{4} \sin ^{4} \beta\right)+\frac{13}{18} g_{s}^{4}\right], \\
\gamma_{3}= & h_{t}^{2} \sin ^{2} \beta T\left(1+\frac{9}{4} g_{w}^{2} \frac{L_{b}}{16 \pi^{2}}-3 h_{t}^{2} \sin ^{2} \frac{L_{f}}{16 \pi^{2}}+4 g_{s}^{2} \frac{L_{b}}{16 \pi^{2}}\right) \\
& -T\left[\frac { L _ { b } } { 1 6 \pi ^ { 2 } } \left(\frac{4}{3} h_{t}^{2} \sin ^{2} \beta g_{s}^{2}+2 h_{t}^{4} \sin ^{4} \beta+6 \lambda h_{t}^{2} \sin ^{2} \beta\right.\right. \\
& \left.+h_{t}^{2}\left(2 \lambda_{3}+\lambda_{4}+h_{t}^{2} \sin ^{2} \beta\right)\right], \\
& \\
\Lambda_{3}^{Q}= & \lambda_{3} T\left(1+\frac{9}{2} g_{w}^{2} \frac{L_{b}}{16 \pi^{2}}-3 h_{t}^{2} \sin ^{2} \frac{L_{f}}{16 \pi^{2}}+4 g_{s}^{2} \frac{L_{b}}{16 \pi^{2}}\right) \\
& -T\left[\frac { L _ { b } } { 1 6 \pi ^ { 2 } } \left(\frac{9}{8} g_{w}^{4}+\frac{4}{3} g_{s}^{2} \lambda_{3}+6 \lambda \lambda_{3}+6 \lambda_{1} \lambda_{3}+2 \lambda_{3}^{2}+2 \lambda \lambda_{4}\right.\right. \\
& +2 \lambda_{1} \lambda_{4}+\lambda_{4}^{2} \cos ^{4} \beta+h_{t}^{4} \sin ^{2} \beta+2 \lambda h_{t}^{2} \sin ^{2} \beta+2 \lambda_{1} h_{t}^{2} \sin ^{2} \beta \\
& \left.\left.+h_{t}^{4} \sin ^{4} \beta+2 h_{t}^{2} \lambda_{4} \sin ^{4} \beta+\lambda_{4}^{2} \sin ^{4} \beta\right)-\frac{L_{f}}{16 \pi^{2}} 2 h_{t}^{4} \sin ^{4} \beta\right],
\end{aligned}
$$

\footnotetext{
${ }^{5}$ For contributions arising from the rest of the supersymmetric particles and the inclusion of Yukawa couplings for the other (s)quarks see ref. 26].
} 


$$
\begin{aligned}
& \Lambda_{4}^{c}=\lambda_{4} \cos ^{2} \beta T\left(1+\frac{9}{2} g_{w}^{2} \frac{L_{b}}{16 \pi^{2}}-3 h_{t}^{2} \sin ^{2} \frac{L_{f}}{16 \pi^{2}}+4 g_{s}^{2} \frac{L_{b}}{16 \pi^{2}}\right) \\
& -T \frac{L_{b}}{16 \pi^{2}}\left(2 \lambda \lambda_{4} \cos ^{2} \beta+2 \lambda_{1} \lambda_{4} \cos ^{2} \beta+g_{w}^{2} \lambda_{4} \cos ^{2} \beta+2 \lambda_{4}^{2} \cos ^{4} \beta\right. \\
& \left.+4 \lambda_{3} \lambda_{4} \cos ^{2} \beta+\frac{4}{3} g_{s}^{2} \lambda_{4} \cos ^{2} \beta\right) \text {, } \\
& \Lambda_{4}^{s}=\lambda_{4} \sin ^{2} \beta T\left(1+\frac{9}{2} g_{w}^{2} \frac{L_{b}}{16 \pi^{2}}-3 h_{t}^{2} \sin ^{2} \frac{L_{f}}{16 \pi^{2}}+4 g_{s}^{2} \frac{L_{b}}{16 \pi^{2}}\right) \\
& -\frac{T}{2} \frac{L_{b}}{16 \pi^{2}}\left(\frac{4}{3} g_{s}^{2}\left(h_{t}^{2}+\lambda_{4}\right) \sin ^{2} \beta+2 \lambda \lambda_{4} \sin ^{2} \beta+2 \lambda_{1} \lambda_{4} \sin ^{2} \beta+2 \lambda_{4}^{2} \sin ^{4} \beta\right. \\
& +2 h_{t}^{4} \sin ^{4} \beta+2 \lambda h_{t}^{2} \sin ^{2} \beta+2 \lambda_{1} h_{t}^{2} \sin ^{2} \beta \\
& +h_{t}^{4} \sin ^{4} \beta+4 h_{t}^{2} \lambda_{4} \sin ^{4} \beta+g_{w}^{2}\left(h_{t}^{2}+\lambda_{4}\right) \sin ^{2} \beta+2 \lambda_{4}^{2} \sin ^{4} \beta \\
& \left.+4 \lambda_{3} \lambda_{4} \sin ^{2} \beta+4 h_{t}^{2} \lambda_{3} \sin ^{2} \beta\right) \text {, } \\
& h_{t}^{L}=h_{t}^{2} \sin ^{2} \beta T\left(1+\frac{9}{2} g_{w}^{2} \frac{L_{b}}{16 \pi^{2}}-3 h_{t}^{2} \sin ^{2} \frac{L_{f}}{16 \pi^{2}}+4 g_{s}^{2} \frac{L_{b}}{16 \pi^{2}}\right) \\
& -\frac{T}{2} \frac{L_{b}}{16 \pi^{2}}\left(\frac{4}{3} g_{s}^{2}\left(h_{t}^{2}+\lambda_{4}\right) \sin ^{2} \beta+2 \lambda \lambda_{4} \sin ^{2} \beta+2 \lambda_{1} \lambda_{4} \sin ^{2} \beta+2 \lambda_{4}^{2} \sin ^{4} \beta\right. \\
& +2 h_{t}^{4} \sin ^{4} \beta+2 \lambda h_{t}^{2} \sin ^{2} \beta+2 \lambda_{1} h_{t}^{2} \sin ^{2} \beta \\
& +h_{t}^{4} \sin ^{4} \beta+4 h_{t}^{2} \lambda_{4} \sin ^{4} \beta+g_{w}^{2}\left(h_{t}^{2}+\lambda_{4}\right) \sin ^{2} \beta+2 \lambda_{4}^{2} \sin ^{4} \beta \\
& \left.+4 \lambda_{3} \lambda_{4} \sin ^{2} \beta+4 h_{t}^{2} \lambda_{3} \sin ^{2} \beta\right) \text {. }
\end{aligned}
$$

We include the relations for the couplings among the heavy fields which will be integrated out at the second stage. These relations are needed only when the 2-loop contribution from these fields to the effective potential are included, see section 2.4. We obtain

$$
\begin{aligned}
g_{s_{1}}^{Q U} & =-\frac{1}{2} g_{s}^{2} T\left(1+\left(\frac{9}{4} g_{w}^{2}+8 g_{s}^{2}\right) \frac{L_{b}}{16 \pi^{2}}\right)-\frac{T}{2}\left(\frac { L _ { b } } { 1 6 \pi ^ { 2 } } \left(3 h_{t}^{4}+\frac{5}{4} g_{s}^{4}\right.\right. \\
& \left.\left.-\frac{7}{6} h_{t}^{2} g_{s}^{2}-\frac{5}{12} g_{s}^{4}+\frac{3}{8} g_{w}^{2}\left(2 h_{t}^{2}-g_{s}^{2}\right)\right)+\frac{5}{6} \frac{g_{s}^{4}}{16 \pi^{2}}\right),
\end{aligned}
$$




$$
\begin{aligned}
& h_{t}^{Q U}=h_{t}^{2} T\left(1+\left(\frac{9}{4} g_{w}^{2}+8 g_{s}^{2}\right) \frac{L_{b}}{16 \pi^{2}}\right)-\frac{T}{2}\left(\frac { L _ { b } } { 1 6 \pi ^ { 2 } } \left(3 h_{t}^{4}+\frac{5}{4} g_{s}^{4}\right.\right. \\
& \left.\left.-\frac{7}{6} h_{t}^{2} g_{s}^{2}-\frac{5}{12} g_{s}^{4}+\frac{3}{8} g_{w}^{2}\left(2 h_{t}^{2}-g_{s}^{2}\right)\right)+\frac{5}{6} \frac{g_{s}^{4}}{16 \pi^{2}}\right) \text {, } \\
& g_{s_{2}}^{Q U}=\frac{1}{6} g_{s}^{2} T\left(1+\left(\frac{9}{4} g_{w}^{2}+8 g_{s}^{2}\right) \frac{L_{b}}{16 \pi^{2}}\right)-T\left(\frac { L _ { b } } { 1 6 \pi ^ { 2 } } \left(h_{t}^{4}+g_{s}^{2} \lambda_{1}\right.\right. \\
& -\frac{1}{2} h_{t}^{2} g_{s}^{2}+\frac{7}{12} g_{s}^{4}+2 h_{t}^{2} \lambda_{3} \sin ^{2} \beta+h_{t}^{2} \lambda_{4} \sin ^{2} \beta \cos ^{2} 2 \beta \\
& \left.\left.+\frac{11}{12} g_{s}^{4}+h_{t}^{4} \sin ^{4} \beta+h_{t}^{2} \lambda_{4} \sin ^{4} \beta\right)+\frac{11}{18} \frac{g_{s}^{4}}{16 \pi^{2}}\right) \\
& g_{s_{1}}^{U D}=\frac{1}{2} g_{s}^{2} T\left(1+8 g_{s}^{2} \frac{L_{b}}{16 \pi^{2}}\right)-T\left(\frac{L_{b}}{16 \pi^{2}}\left(-h_{t}^{2} g_{s}^{2}+\frac{5}{2} g_{s}^{4}\right)+\frac{5}{6} \frac{g_{s}^{4}}{16 \pi^{2}}\right) \\
& g_{s_{2}}^{U D}=-\frac{1}{6} g_{s}^{2} T\left(1+8 g_{s}^{2} \frac{L_{b}}{16 \pi^{2}}\right) \\
& -T\left(\frac{L_{b}}{16 \pi^{2}}\left(\frac{1}{3} h_{t}^{2} g_{s}^{2}+\frac{1}{36} g_{s}^{4}+\frac{11}{12} g_{s}^{4}\right)+\frac{11}{18} \frac{g_{s}^{4}}{16 \pi^{2}}\right) \text {, } \\
& g_{s_{1}}^{Q D}=-\frac{1}{2} g_{s}^{2} T\left(1+\left(\frac{9}{4} g_{w}^{2}+8 g_{s}^{2}\right) \frac{L_{b}}{16 \pi^{2}}\right)-T\left(\frac { L _ { b } } { 1 6 \pi ^ { 2 } } \left(h_{t}^{2} g_{s}^{2}+\frac{5}{4} g_{s}^{4}\right.\right. \\
& \left.\left.-\frac{5}{12} g_{s}^{4}+\frac{3}{8} g_{w}^{2}\left(2 h_{t}^{2}-g_{s}^{2}\right)\right)+\frac{5}{6} \frac{g_{s}^{4}}{16 \pi^{2}}\right) \\
& g_{s 2}^{Q D}=\frac{1}{6} g_{s}^{2} T\left(1+\left(\frac{9}{4} g_{w}^{2}+8 g_{s}^{2}\right) \frac{L_{b}}{16 \pi^{2}}\right)-T\left(\frac { L _ { b } } { 1 6 \pi ^ { 2 } } \left(-\frac{1}{6} h_{t}^{2} g_{s}^{2}+\frac{11}{12} g_{s}^{4}\right.\right. \\
& \left.\left.+\frac{7}{12} g_{s}^{4}+g_{s}^{2} \lambda_{1}+\frac{3}{8} g_{w}^{2}\left(2 h_{t}^{2}-g_{s}^{2}\right)\right)+\frac{11}{18} \frac{g_{s}^{4}}{16 \pi^{2}}\right)
\end{aligned}
$$




$$
\begin{aligned}
& \Lambda_{1}=\lambda_{1} T\left(1+\left(\frac{9}{2} g_{w}^{2}+8 g_{s}^{2}\right) \frac{L_{b}}{\left(16 \pi^{2}\right)}\right)-\frac{T}{2}\left(\frac { L _ { b } } { ( 1 6 \pi ^ { 2 } ) } \left(h_{t}^{4}-\frac{2}{3} h_{t}^{2} g_{s}^{2}\right.\right. \\
& +\frac{23}{18} g_{s}^{4}+\frac{5}{16} g_{w}^{4}+\frac{3}{4} g_{s}^{2} g_{w}^{2}+2 \lambda_{3}^{2}+2 \lambda_{3} \lambda_{4}+\lambda_{4}^{2} \cos ^{4} \beta+2 h_{t}^{2} \lambda_{3} \sin ^{2} \beta \\
& \left.\left.+h_{t}^{4} \sin ^{4} \beta+2 h_{t}^{2} \lambda_{4} \sin ^{4} \beta+\lambda_{4}^{2} \sin ^{4} \beta+\frac{13}{12} g_{s}^{4}\right)+\frac{13}{18} g_{s}^{4}\right) \\
& \lambda_{Q_{3}}=\frac{g_{s}^{2}}{6} T\left(1+\left(\frac{9}{2} g_{w}^{2}+8 g_{s}^{2}\right) \frac{L_{b}}{\left(16 \pi^{2}\right)}\right)-\frac{T}{2}\left(\frac { L _ { b } } { ( 1 6 \pi ^ { 2 } ) } \left(h_{t}^{4}-\frac{2}{3} h_{t}^{2} g_{s}^{2}\right.\right. \\
& +\frac{23}{18} g_{s}^{4}+\frac{5}{16} g_{w}^{4}+\frac{3}{4} g_{s}^{2} g_{w}^{2}+2 \lambda_{3}^{2}+2 \lambda_{3} \lambda_{4}+\lambda_{4}^{2} \cos ^{4} \beta+2 h_{t}^{2} \lambda_{3} \sin ^{2} \beta+h_{t}^{4} \sin ^{4} \beta \\
& \left.\left.+2 h_{t}^{2} \lambda_{4} \sin ^{4} \beta+\lambda_{4}^{2} \sin ^{4} \beta+\frac{13}{12} g_{s}^{4}\right)+\frac{13}{18} g_{s}^{4}\right), \\
& g_{s_{1}}^{Q Q}=\frac{g_{s}^{2}}{6} T\left(1+\left(\frac{9}{2} g_{w}^{2}+8 g_{s}^{2}\right) \frac{L_{b}}{\left(16 \pi^{2}\right)}\right)-T\left(\frac { L _ { b } } { ( 1 6 \pi ^ { 2 } ) } \left(h_{t}^{4}-h_{t}^{2} g_{s}^{2}\right.\right. \\
& +\frac{11}{12} g_{s}^{4}+\frac{5}{4} g_{s}^{4}+\lambda_{4}^{2} \cos ^{2} \beta-2 h_{t}^{2} \lambda_{4} \sin ^{2} \beta \cos ^{2} \beta-2 \lambda_{4}^{2} \sin ^{2} \beta \cos ^{2} \beta+h_{t}^{4} \\
& \left.\left.+2 h_{t}^{2} \lambda_{4} \sin ^{4} \beta+\lambda_{4}^{2} \sin ^{2} \beta+g_{s}^{2} g_{w}^{2}-\frac{3}{16} g_{w}^{4}\right)+\frac{5}{6} \frac{g_{s}^{4}}{\left(16 \pi^{2}\right)}\right) \\
& g_{s_{2}}^{Q Q}=\frac{g_{s}^{2}}{6} T\left(1+\left(\frac{9}{2} g_{w}^{2}+8 g_{s}^{2}\right) \frac{L_{b}}{\left(16 \pi^{2}\right)}\right)-\frac{T}{2}\left(\frac { L _ { b } } { ( 1 6 \pi ^ { 2 } ) } \left(\frac{1}{3} h_{t}^{2} g_{s}^{2}+\frac{1}{36} g_{s}^{4}\right.\right. \\
& +\frac{11}{12} g_{s}^{4}+2 \lambda_{3}^{2}+2 \lambda_{3} \lambda_{4}+2 h_{t}^{2} \lambda_{3} \sin ^{2} \beta+2 h_{t}^{2} \lambda_{4} \sin ^{2} \beta \cos ^{2} \beta \\
& \left.\left.+2 \lambda_{4}^{2} \sin ^{2} \beta \cos ^{2} \beta+\frac{1}{8} g_{w}^{4}-\frac{1}{4} g_{s}^{2} g_{w}^{2}\right)+\frac{11}{18} \frac{g_{s}^{4}}{\left(16 \pi^{2}\right)}\right) \text {. }
\end{aligned}
$$

A few technical comments are in order. We point out that the full one-loop contribution to the quartic coupling $\left|\epsilon_{i j} H^{i} Q^{j}\right|^{2}$ is given by the sum of eqs. (14) and (15). Similarly, for the quartic coupling $Q_{i \alpha}^{*} U_{\alpha}^{*} Q_{i \beta} U_{\beta}$ the full contributions arises from eqs. (16) and (17). It is also important 
to note that there are off-diagonal (in colour space) gluonic contributions to the quartic couplings involving the strong gauge coupling.

Additionally, when the full supersymmetric spectrum is not included then the running of each of the strong quartic couplings given in eqs. (16)-(26) is different. Although the gluino contribution is decoupled under our assumptions, we now write as a check the gluino contributions to the logarithmic part of the quartic couplings. This shows how, if one includes the full spectrum, then the relation between the beta-function coefficients is the same as the relation between the couplings.

The gluino contribution, in units of $T \frac{L_{f}}{16 \pi^{2}} \frac{1}{3} g_{s}^{4}$, to the self-couplings is $\frac{22}{3}$. Using the same units, for $g_{s_{1}}^{Q U}, g_{s_{1}}^{Q D}$ the contribution is -4 , for $g_{s_{2}}^{Q U}, g_{s_{2}}^{Q D}$ the contribution is $\frac{20}{3}$, for $g_{s_{1}}^{U D}, g_{s_{1}}^{Q Q}$ the additional term is 14 , and for $g_{s_{2}}^{U D}, g_{s_{2}}^{Q Q}$ the contribution is $\frac{2}{3}$.

\subsection{Second Stage}

Another simplification of the effective theory can be obtained by integrating out the scalar fields which are massive at the transition point. As we have seen the static modes corresponding to the scalar fields $Q, D, A_{o}, C_{o}$, acquired thermal masses proportional to $\sim g_{w(s)} T$, as a consequence of the integration out of the non-zero Matsubara modes. The second stage proceeds in exactly the same way as in reference [14]. We include the additional corrections arising from the couplings we have considered.

\subsubsection{Couplings}

The final expression for the tree level 3D potential is given by

$$
V_{3 D}=\bar{m}_{H_{3}}^{2} H^{\dagger} H+\bar{\lambda}_{H_{3}}\left(H^{\dagger} H\right)^{2}+\bar{m}_{U_{3}}^{2} U^{\dagger} U+\bar{\lambda}_{U_{3}}\left(U^{\dagger} U\right)^{2}+\bar{\gamma}_{3} H^{\dagger} H U^{\dagger} U
$$

where the scalar couplings are now

$$
\begin{aligned}
\bar{\lambda}_{H_{3}} & =\lambda_{H_{3}}-\frac{3}{16} \frac{g_{w_{3}}^{4}}{8 \pi m_{A_{0}}}-\frac{3}{8 \pi m_{Q_{3}}}\left(\Lambda_{3}^{2}+\Lambda_{3}\left(\Lambda_{4}^{c}+\Lambda_{4}^{s}\right)\right. \\
& \left.+\frac{1}{2}\left(\left(\Lambda_{4}^{c}\right)^{2}+\left(\Lambda_{4}^{s}\right)^{2}\right)+h_{t}^{L} \Lambda_{3}^{Q}+h_{t}^{L} \Lambda_{4}^{s}+\frac{1}{2}\left(h_{t}^{L}\right)^{2}\right)
\end{aligned}
$$




$$
\begin{aligned}
\bar{\lambda}_{U_{3}} & =\lambda_{U_{3}}-\frac{13}{36} \frac{g_{s_{3}}^{4}}{8 \pi m_{C_{0}}}-\frac{1}{8 \pi m_{D_{3}}}\left(\frac{1}{2}\left(g_{s_{1}}^{U D}+g_{s_{2}}^{U D}\right)^{2}+\left(g_{s_{2}}^{U D}\right)^{2}\right) \\
& -\frac{1}{8 \pi m_{Q_{3}}}\left(\left(h_{t}^{Q U}\right)^{2}-2 h_{t}^{Q U} g_{s_{1}}^{Q U}+2 h_{t}^{Q U} g_{s_{2}}^{Q U}+\left(g_{s_{1}}^{U D}+g_{s_{2}}^{U D}\right)^{2}+2\left(g_{s_{2}}^{U D}\right)^{2}\right) \\
& \gamma_{3}=\gamma_{3}-\frac{1}{8 \pi m_{Q_{3}}}\left(h_{t}^{Q U}+g_{s_{1}}^{Q U}+3 g_{s_{2}}^{Q U}\right)\left(2 \Lambda_{3}^{Q}+\Lambda_{4}^{c}+\Lambda_{4}^{s}+h_{t}^{L}\right) .
\end{aligned}
$$

The 3D gauge couplings which appear in the $\mathrm{SU}(2)$ and $\mathrm{SU}(3)$ covariant derivatives of the effective theory are

$$
\begin{gathered}
\bar{g}_{w_{3}}^{2}=g_{w_{3}}^{2}\left(1-\frac{g_{w_{3}}^{2}}{24 \pi m_{A_{0}}}-\frac{g_{w_{3}}^{2}}{16 \pi m_{Q_{3}}}\right), \\
\bar{g}_{s_{3}}^{2}=g_{s_{3}}^{2}\left(1-\frac{g_{s_{3}}^{2}}{16 \pi m_{C_{0}}}-\frac{g_{s_{3}}^{2}}{24 \pi m_{Q_{3}}}-\frac{g_{s_{3}}^{2}}{48 \pi m_{D_{3}}}\right) .
\end{gathered}
$$

\subsubsection{Mass terms}

The one-loop contribution to the mass terms can be obtained directly as shown in ref. [21]:

$$
\begin{aligned}
\bar{m}_{H_{3}}^{2} & =m_{H_{3}}^{2}-\frac{3}{16 \pi} g_{w_{3}} m_{A_{0}}-\frac{3}{4 \pi}\left(2 \Lambda_{3}^{Q}+\Lambda_{4}^{c}+\Lambda_{4}^{s}+h_{t}^{L}\right) m_{Q_{3}} \\
\bar{m}_{U_{3}}^{2} & =m_{U_{3}}^{2}-\frac{1}{3 \pi} g_{s_{3}} m_{C_{0}}-\frac{1}{4 \pi}\left(2 h_{t}^{Q U}+2 g_{s_{1}}^{Q U}+6 g_{s_{2}}^{Q U}\right) m_{Q_{3}} \\
& -\frac{1}{4 \pi}\left(2 g_{s_{1}}^{U D}+6 g_{s_{2}}^{U D}\right) m_{D_{3}} .
\end{aligned}
$$

Until now, our procedure has been exactly the same as in previous 3D reductions of the MSSM. However, in order to precisely fix the scales of the couplings that appear in the thermal polarizations of eqs. (2)-(3), one needs to perform a 2-loop evaluation of the effective potential. In their 
calculation, Bodeker et al. 14 took the values of the couplings in the screening parts of the 3D masses to be equal to the $3 \mathrm{D}$ values of these couplings. This is the correct result when two-loop corrections are included [21, 29], see eqs. (67) and (68) below. In addition, the mass parameters are renormalized in the $3 \mathrm{D}$ theory,

$$
\begin{gathered}
\bar{m}_{H_{3}}^{2}(\mu)=\bar{m}_{H_{3}}^{2}+\frac{1}{\left(16 \pi^{2}\right)} f_{2 m_{H}} \log \frac{\Lambda_{H_{3}}}{\mu}, \\
\bar{m}_{U_{3}}^{2}(\mu)=\bar{m}_{U_{3}}^{2}+\frac{1}{\left(16 \pi^{2}\right)} f_{2 m_{U}} \log \frac{\Lambda_{U_{3}}}{\mu} .
\end{gathered}
$$

The expressions for the 2-loop beta functions $f_{2 m_{H}}, f_{2 m_{U}}$ for the mass parameters have been given in ref. [14]. As mentioned there in order to fix the values of the parameters $\Lambda_{H_{3}}$ and $\Lambda_{U_{3}}$ we

must employ the 2-loop effective potential of the 4D theory. In refs. [14, 30] an estimate of $\Lambda_{H_{3}} \sim \Lambda_{U_{3}} \sim 7 T$ was used. The expressions for the 2-loop effective potential in a $H$ ( $\phi$-direction) and $U$ ( $\chi$-direction $)$ background have been given for 4D in the paper by Carena et al. [15, and for $3 \mathrm{D}$ by Bodeker et al. [24]. In sections 2.3 and 2.4 we perform a two-loop calculation of the effective potential, incorporating all of the corrections to the 3D couplings obtained in the previous sections, to determine the exact values of $\Lambda_{H_{3}}$ and $\Lambda_{U_{3}}$.

We will analyse the effect on the critical temperatures when these corrections are included. Qualitatively we can say that if the net effect increases the value of $\Lambda_{H_{3}}$, then the critical temperature in the $\phi$-direction decreases, and vice versa. A similar effect occurs in the $\chi$-direction. Thus, as the range of values of $m_{\tilde{t}_{R}}$, which has been determined to give rise to a two-stage phase transition, is small, a more precise evaluation of the critical temperatures is of interest. This could have the effect of either reducing or enhancing the allowed range of values of the right stop mass, $m_{\tilde{t}_{R}}$, for which a two-stage phase transition can occur.

\subsection{Two-loop contributions}

The strategy we employ follows that of ref. [21]. The idea is to use the 4D 2-loop effective potential in order to fix the scales in the 3D theory, and to use the 3D effective potential expressions for the Higgs and stop fields given in ref. [14] to analyse the phase transition. We calculate the unresummed 
2-loop effective potential in order to include all 4D corrections to the mass parameters, resummation is automatically included in the calculation of the 2-loop effective potential in the 3D theory. We must also include the contributions to the 2-loop effective potential of the static modes, which have been integrated out at the second stage (includes the effects of resummation of the heavy fields).

There are several effects that must be considered in order to obtain all of the contributions (constant and logarithmic) to the mass parameters. From the $4 \mathrm{D}$ effective potential one finds the two-loop contributions from the gauge bosons, Higgs, right handed stop, left handed squark doublet, right handed sbottom, and top quark. The expression for the 2-loop effective potential can be found in refs. [12, 14, 15]. In particular, within our approximations for the $\phi$-direction, the appropriate expression is that given in ref. [12], because we are including the 3rd generation squark doublet and the right handed sbottom in the thermal bath $\mathrm{f}$. The main difference is that the $D$ functions appearing below correspond to the unresummed expressions. Additionally we must include the effects arising at the second stage of reduction from the left handed squark doublet, the right handed sbottom, the scalar triplet and the scalar octet. We now derive the effective potential at finite temperature using the background fields $\phi$ and $\chi=\tilde{t}_{R \alpha} u^{\alpha}$, where we have chosen the unit vector in colour space $u^{\alpha}=(1,0,0)$. We now write the expressions in the shifted theory of the mass spectrum after the first stage of integration. The gauge bosons masses are

$$
m_{W, Z}^{2}=\frac{1}{4} g_{w}^{2} \phi^{2}, \quad m_{G}^{2}=\frac{1}{4} g_{s}^{2} \chi^{2}, \quad \bar{m}_{G}^{2}=\frac{4}{3} m_{G}^{2} .
$$

With no mixing in the Higgs sector, the Goldstone bosons and Higgs masses are

$$
\begin{aligned}
& m_{\pi}^{2}=m_{H}^{2}+\lambda \phi^{2}+h_{t}^{2} \sin ^{2} \beta \frac{\chi^{2}}{2}, \\
& m_{h}^{2}=m_{H}^{2}+3 \lambda \phi^{2}+h_{t}^{2} \sin ^{2} \beta \frac{\chi^{2}}{2}, \\
& m_{\omega}^{2}=m_{\bar{\omega}}^{2}=m_{U}^{2}+\lambda_{U} \chi^{2}+h_{t}^{2} \sin ^{2} \beta \frac{\phi^{2}}{2}, \\
& m_{u}^{2}=m_{U}^{2}+3 \lambda \chi^{2}+h_{t}^{2} \sin ^{2} \beta \frac{\phi^{2}}{2} .
\end{aligned}
$$

\footnotetext{
${ }^{6}$ For most of our analysis we will include the third-generation left squark doublet in the thermal bath.
} 
The masses of the rest of the scalars contributing to the effective potential are given by

$$
\begin{gathered}
m_{\tilde{t}_{L_{1}}}^{2}=m_{Q}^{2}+\left(h_{t}^{2} \sin ^{2} \beta+\lambda_{3}+\lambda_{4} \sin ^{2} \beta\right) \frac{\phi^{2}}{2}+\left(h_{t}^{2}-\frac{g_{s}^{2}}{3}\right) \frac{\chi^{2}}{2} \\
m_{\tilde{t}_{L_{2,3}}}^{2}=m_{Q}^{2}+\left(h_{t}^{2} \sin ^{2} \beta+\lambda_{3}+\lambda_{4} \sin ^{2} \beta\right) \frac{\phi^{2}}{2}+\left(\frac{g_{s}^{2}}{6}\right) \frac{\chi^{2}}{2} \\
m_{\tilde{b}_{L_{1}}}^{2}=m_{Q}^{2}+\left(\lambda_{3}+\lambda_{4} \cos ^{2} \beta\right) \frac{\phi^{2}}{2}+\left(h_{t}^{2}-\frac{g_{s}^{2}}{3}\right) \frac{\chi^{2}}{2} \\
m_{\tilde{b}_{L_{2,3}}}^{2}=m_{Q}^{2}+\left(\lambda_{3}+\lambda_{4} \cos ^{2} \beta\right) \frac{\phi^{2}}{2}+\left(\frac{g_{s}^{2}}{6}\right) \frac{\chi^{2}}{2} \\
m_{\tilde{b}_{R_{1}}}^{2}=m_{D}^{2}+\left(\frac{g_{s}^{2}}{6}\right) \frac{\chi^{2}}{2} \\
m_{\tilde{b}_{R_{2,3}}^{2}}^{2}=m_{D}^{2}-\left(\frac{g_{s}^{2}}{3}\right) \frac{\chi^{2}}{2} .
\end{gathered}
$$

\subsection{1 $\phi$-direction}

The contributions to the terms in $m_{H_{3}}^{2}(\mu)$ proportional to $g_{w}^{4}, g_{w}^{2} \lambda_{H_{3}}$ and $\lambda_{H_{3}}^{2}$ from Standard Model particles have been calculated in the paper by Kajantie et al. [21]. The correct expression is obtained from eq. (151) of [21], substituting $\lambda=\frac{1}{8}\left(g_{w}^{2}+g^{\prime 2}\right) \cos 2 \beta$ and $g_{Y}^{2}=h_{t}^{2} \sin ^{2} \beta$. For the Standard Model, this already includes the finite contributions from counterterms.

The additional corrections that arise from supersymmetric particles can be calculated using the 2-loop unresummed potential. The expressions in the integral form have been given in ref. [12] for zero squark mixing, and we include them for completeness. Our notation for the $D$ functions corresponds to that of ref. [21]. The contributions from the 2-loop graphs containing supersymmetric particles are given below. For the $\phi$-direction, we can drop the colour index of the squark masses: 


$$
\begin{aligned}
& (S S V)=-\frac{g_{w}^{2}}{8} N_{c}\left[D_{S S V}\left(m_{\tilde{t}_{L}}, m_{\tilde{t}_{L}}, m_{W}\right)+D_{S S V}\left(m_{\tilde{b}_{L}}, m_{\tilde{b}_{L}}, m_{W}\right)+4 D_{S S V}\left(m_{\tilde{t}_{L}}, m_{\tilde{b}_{L}}, m_{W}\right)\right] \\
& -\frac{g_{s}^{2}}{4}\left(N_{c}^{2}-1\right)\left[D_{S S V}\left(m_{\tilde{t}_{L}}, m_{\tilde{t}_{L}}, 0\right)+D_{S S V}\left(m_{\tilde{b}_{L}}, m_{\tilde{b}_{L}}, 0\right)\right. \\
& \left.+D_{S S V}\left(m_{\tilde{t}_{R}}, m_{\tilde{t}_{R}}, 0\right)+D_{S S V}\left(m_{\tilde{b}_{R}}, m_{\tilde{b}_{R}}, 0\right)\right], \\
& (S S S)=-N_{c}\left[\left(h_{t}^{2} \sin ^{2} \beta+\frac{g_{w}^{2}}{4} \cos 2 \beta\right)^{2} D_{S S S}\left(m_{\tilde{t}_{L}}, m_{\tilde{t}_{L}}, m_{h}\right)+\left(\frac{g_{w}^{2}}{4} \cos 2 \beta\right)^{2} D_{S S S}\left(m_{\tilde{b}_{L}}, m_{\tilde{b}_{L}}, m_{h}\right)\right. \\
& \left.+\left(h_{t}^{2} \sin ^{2} \beta\right)^{2} D_{S S S}\left(m_{\tilde{t}_{R}}, m_{\tilde{t}_{R}}, m_{h}\right)+\left(h_{t}^{2} \sin ^{2} \beta+\frac{g_{w}^{2}}{2} \cos 2 \beta\right)^{2} D_{S S S}\left(m_{\tilde{t}_{L}}, m_{\tilde{b}_{L}}, m_{\pi}\right)\right] \frac{\phi^{2}}{2},(46) \\
& (S V)=-\frac{1}{4} g_{s}^{2}\left(N_{c}^{2}-1\right)\left[D_{S V}\left(m_{\tilde{t}_{L}}, 0\right)+D_{S V}\left(m_{\tilde{b}_{L}}, 0\right)+D_{S V}\left(m_{\tilde{t}_{R}}, 0\right)+D_{S V}\left(m_{\tilde{b}_{R}}, 0\right)\right] \\
& -\frac{3}{8} g_{w}^{2} N_{c}\left[D_{S V}\left(m_{\tilde{t}_{L}}, m_{W}\right)+D_{S V}\left(m_{\tilde{b}_{L}}, m_{W}\right)\right], \\
& (S S)=\frac{g_{w}^{2}}{4} N_{c}\left(2-N_{c}\right) D_{S S}\left(m_{\tilde{t}_{L}}, m_{\tilde{b}_{L}}\right)+h_{t}^{2} N_{c}\left[D_{S S}\left(m_{\tilde{t}_{L}}, m_{\tilde{t}_{R}}\right)+D_{S S}\left(m_{\tilde{b}_{L}}, m_{\tilde{t}_{R}}\right)\right] \\
& +\left(\frac{g_{w}^{2}}{8}+\frac{g_{s}^{2}}{6}\right) N_{c}\left(N_{c}+1\right)\left[D_{S S}\left(m_{\tilde{t}_{L}}, m_{\tilde{t}_{L}}\right)+D_{S S}\left(m_{\tilde{b}_{L}}, m_{\tilde{b}_{L}}\right)\right] \\
& +\frac{g_{s}^{2}}{6} N_{c}\left(N_{c}+1\right)\left[D_{S S}\left(m_{\tilde{t}_{R}}, m_{\tilde{t}_{R}}\right)+D_{S S}\left(m_{\tilde{b}_{R}}, m_{\tilde{b}_{R}}\right)\right] \\
& +N_{c}\left(\frac{1}{2} h_{t}^{2} \sin ^{2} \beta+\frac{1}{8} g_{w}^{2} \cos 2 \beta\right)\left[D_{S S}\left(m_{\tilde{t}_{L}}, m_{h}\right)+2 D_{S S}\left(m_{\tilde{b}_{L}}, m_{\pi}\right)+D_{S S}\left(m_{\tilde{t}_{L}}, m_{\pi}\right)\right] \\
& -\frac{1}{8} N_{c} g_{w}^{2} \cos 2 \beta\left[D_{S S}\left(m_{\tilde{b}_{L}}, m_{h}\right)+2 D_{S S}\left(m_{\tilde{t}_{L}}, m_{\pi}\right)+D_{S S}\left(m_{\tilde{b}_{L}}, m_{\pi}\right)\right] \\
& +\frac{1}{2} N_{c} h_{t}^{2} \sin ^{2} \beta\left[D_{S S}\left(m_{\tilde{t}_{R}}, m_{h}\right)+3 D_{S S}\left(m_{\tilde{t}_{R}}, m_{\pi}\right)\right] \text {. }
\end{aligned}
$$

There are also counterterm contributions to the mass terms; for the $\phi$-direction they correspond to eq. (B.3) of ref. 12.

$$
\delta V=-\frac{T^{2}}{16 \pi^{2}} \frac{\phi^{2}}{96}\left(3 g_{w}^{4}+\frac{11}{9} g^{\prime 4}\right)
$$




\subsection{2 $\chi$-direction}

The two-loop unresummed effective potential in the $\chi$-direction is given by the following contributions ${ }^{7}$, 踝:

$$
\begin{aligned}
( & V V V)=-g_{s}^{2} \frac{N_{c}}{4}\left[\left(N_{c}-2\right) D_{V V V}\left(m_{G}, m_{G}, 0\right)+D_{V V V}\left(m_{G}, m_{G}, m_{G}\right)\right] \\
& (\eta \eta V)=-g_{s}^{2} \frac{N_{c}}{2}\left[2\left(N_{c}-1\right) D_{\eta \eta V}\left(0,0, m_{G}\right)+D_{\eta \eta V}\left(0,0, \bar{m}_{G}\right)\right] \\
(V V)=- & g_{s}^{2} \frac{N_{c}}{8}\left[2\left(N_{c}-2\right) D_{V V}\left(0, m_{G}\right)+2 D_{V V}\left(m_{G}, \bar{m}_{G}\right)+\left(N_{c}-1\right) D_{V V}\left(m_{G}, m_{G}\right)\right] \\
(S S V)= & -\frac{g_{w}^{2}}{8}\left[D_{S S V}\left(m_{\tilde{t}_{L 1}}, m_{\tilde{t}_{L 1}}, 0\right)+D_{S S V}\left(m_{\tilde{t}_{L 2}}, m_{\tilde{t}_{L 2}}, 0\right)+D_{S S V}\left(m_{\tilde{t}_{L 3}}, m_{\tilde{t}_{L 3}}, 0\right)\right. \\
& +D_{S S V}\left(m_{\tilde{b}_{L 1}}, m_{\tilde{b}_{L 1}}, 0\right)+D_{S S V}\left(m_{\tilde{b}_{L 2}}, m_{\tilde{b}_{L 2}}, 0\right)+D_{S S V}\left(m_{\tilde{b}_{L 3}}, m_{\tilde{b}_{L 3}}, 0\right) \\
& \left.+4\left(D_{S S V}\left(m_{\tilde{t}_{L 1}}, m_{\tilde{b}_{L 1}}, 0\right)+D_{S S V}\left(m_{\tilde{t}_{L 2}}, m_{\tilde{b}_{L 2}}, 0\right)+D_{S S V}\left(m_{\tilde{t}_{L 3}}, m_{\tilde{b}_{L 3}}, 0\right)\right)\right] \\
& -g_{s}^{2} \frac{1}{4}\left[\left(N_{c}-1\right) D_{S S V}\left(m_{\omega}, \bar{m}_{\omega}, m_{G}\right)+\left(N_{c}-1\right) D_{S S V}\left(m_{\omega}, m_{u}, m_{G}\right)\right. \\
& +\frac{N_{c}-1}{N_{c}} D_{S S V}\left(\bar{m}_{\omega}, m_{u}, m_{G}\right)+\frac{1}{N_{c}} D_{S S V}\left(m_{\omega}, m_{\omega}, \bar{m}_{G}\right) \\
& +N_{c}\left(N_{c}-2\right) D_{S S V}\left(m_{\omega}, m_{\omega}, 0\right)+2\left(N_{c}-1\right) D_{S S V}\left(m_{\tilde{t}_{L 1}}, m_{\tilde{t}_{L 2}}, m_{G}\right) \\
& +\frac{N_{c}-1}{N_{c}} D_{S S V}\left(m_{\tilde{t}_{L 1}}, m_{\tilde{t}_{L 1}}, \bar{m}_{G}\right)+\frac{1}{N_{c}} D_{S S V}\left(m_{\tilde{t}_{L 2}}, m_{\tilde{t}_{L 2}}, \bar{m}_{G}\right) \\
& +N_{c}\left(N_{c}-2\right) D_{S S V}\left(m_{\tilde{t}_{L 2}}, m_{\tilde{t}_{L 2}}, 0\right) \\
& +2\left(N_{c}-1\right) D_{S S V}\left(m_{\tilde{b}_{L 1}}, m_{\tilde{b}_{L 2}}, m_{G}\right)+\frac{N_{c}-1}{N_{c}} D_{S S V}\left(m_{\tilde{b}_{L 1}}, m_{\tilde{b}_{L 1}}, \bar{m}_{G}\right) \\
& +\frac{1}{N_{c}} D_{S S V}\left(m_{\tilde{b}_{L 2}}, m_{\tilde{b}_{L 2}}, \bar{m}_{G}\right)+N_{c}\left(N_{c}-2\right) D_{S S V}\left(m_{\tilde{b}_{L 2}}, m_{\tilde{b}_{L 2}}, 0\right)
\end{aligned}
$$

\footnotetext{
${ }^{7}$ The expression given for the $4 \mathrm{D}$ effective potential would correspond to the usual resummed 2-loop $4 \mathrm{D}$ effective potential if we use the resummed expressions in the $D$ functions appearing below.

${ }^{8} \mathrm{As} m_{\tilde{t}_{L 2}}=m_{\tilde{t}_{L 3}}, m_{\tilde{b}_{L 2}}=m_{\tilde{b}_{L 3}}, m_{\tilde{b}_{R 2}}=m_{\tilde{b}_{R 3}}$ in the $\chi$-direction, we just multiply by a factor of 2 the contributions from these fields in some of the following expressions.
} 


$$
\begin{aligned}
& +2\left(N_{c}-1\right) D_{S S V}\left(m_{\tilde{b}_{R 1}}, m_{\tilde{b}_{R 2}}, m_{G}\right)+\frac{N_{c}-1}{N_{c}} D_{S S V}\left(m_{\tilde{b}_{R 1}}, m_{\tilde{b}_{R 1}}, \bar{m}_{G}\right) \\
& \left.+\frac{1}{N_{c}} D_{S S V}\left(m_{\tilde{b}_{R 2}}, m_{\tilde{b}_{R 2}}, \bar{m}_{G}\right)+N_{c}\left(N_{c}-2\right) D_{S S V}\left(m_{\tilde{b}_{R 2}}, m_{\tilde{b}_{R 2}}, 0\right)\right] \\
& (V V S)=-g_{s}^{2} \frac{m_{G}^{2}}{8}\left[\left(N_{c}-1\right) D_{V V S}\left(m_{G}, m_{G}, m_{u}\right)+2 \frac{\left(N_{c}-1\right)^{2}}{N^{2}} D_{V V S}\left(\bar{m}_{G}, \bar{m}_{G}, m_{u}\right)\right. \\
& \left.+N_{c}\left(N_{c}-2\right) D_{V V S}\left(0, m_{G}, m_{\omega}\right)+\frac{\left(N_{c}-2\right)^{2}}{N_{c}} D_{V V S}\left(m_{G}, \bar{m}_{G}, m_{\omega}\right)\right] \\
& (S V)=-\frac{g_{s}^{2}}{8}\left[2 N_{c}\left(N_{c}-2\right) D_{S V}\left(m_{\omega}, 0\right)+\left(N_{c}-1\right)\left[3 D_{S V}\left(m_{\omega}, m_{G}\right)+D_{S V}\left(m_{u}, m_{G}\right)\right]\right. \\
& +\frac{1}{N_{c}}\left[\left(N_{c}+1\right) D_{S V}\left(m_{\omega}, \bar{m}_{G}\right)+\left(N_{c}-1\right) D_{S V}\left(m_{u}, \bar{m}_{G}\right)\right] \\
& +2 N_{c}\left(N_{c}-2\right) D_{S V}\left(m_{\tilde{t}_{L 2}}, 0\right)+\left(N_{c}-1\right)\left[2 D_{S V}\left(m_{\tilde{t}_{L 2}}, m_{G}\right)+2 D_{S V}\left(m_{\tilde{t}_{L 1}}, m_{G}\right)\right] \\
& +\frac{1}{N_{c}}\left[2 D_{S V}\left(m_{\tilde{t}_{L 2}}, \bar{m}_{G}\right)+2 D_{S V}\left(m_{\tilde{t}_{L 1}}, \bar{m}_{G}\right)\right] \\
& +2 N_{c}\left(N_{c}-2\right) D_{S V}\left(m_{\tilde{b}_{L 2}}, 0\right)+\left(N_{c}-1\right)\left[2 D_{S V}\left(m_{\tilde{b}_{L 2}}, m_{G}\right)+2 D_{S V}\left(m_{\tilde{b}_{L 1}}, m_{G}\right)\right] \\
& +\frac{1}{N_{c}}\left[2 D_{S V}\left(m_{\tilde{b}_{L 2}}, \bar{m}_{G}\right)+2 D_{S V}\left(m_{\tilde{b}_{L 1}}, \bar{m}_{G}\right)\right] \\
& +2 N_{c}\left(N_{c}-2\right) D_{S V}\left(m_{\tilde{b}_{R 2}}, 0\right)+\left(N_{c}-1\right)\left[2 D_{S V}\left(m_{\tilde{b}_{R 2}}, m_{G}\right)+2 D_{S V}\left(m_{\tilde{b}_{R 1}}, m_{G}\right)\right] \\
& +\frac{1}{N_{c}}\left[2 D_{S V}\left(m_{\tilde{b}_{R 2}}, \bar{m}_{G}\right)+2 D_{S V}\left(m_{\tilde{b}_{R 1}}, \bar{m}_{G}\right)\right] \\
& -\frac{3}{8} g_{w}^{2}\left[D_{S V}\left(m_{\tilde{t}_{L 1}}, 0\right)+D_{S V}\left(m_{\tilde{t}_{L 2}}, 0\right)+D_{S V}\left(m_{\tilde{t}_{L 3}}, 0\right)\right. \\
& \left.+D_{S V}\left(m_{\tilde{b}_{L 1}}, 0\right)+D_{S V}\left(m_{\tilde{b}_{L 2}}, 0\right)+D_{S V}\left(m_{\tilde{b}_{L 3}}, 0\right)\right] \\
& (S S S)=-\lambda_{U}^{2} \chi^{2}\left[3 D_{S S S}\left(m_{u}, m_{u}, m_{u}\right)+\left(2 N_{c}-1\right) D_{S S S}\left(m_{u}, m_{\omega}, m_{\omega}\right)\right] \\
& -\chi^{2}\left[\frac{1}{4} h_{t}^{2} \sin ^{2} \beta\left[D_{S S S}\left(m_{u}, m_{h}, m_{h}\right)+3 D_{S S S}\left(m_{u}, m_{\pi}, m_{\pi}\right)\right]\right. \\
& -\frac{\chi^{2}}{2}\left[\left(h_{t}^{2}-\frac{1}{3} g_{s}^{2}\right)^{2} D_{S S S}\left(m_{u}, m_{\tilde{t}_{L 1}}, m_{\tilde{t}_{L 1}}\right)\right.
\end{aligned}
$$




$$
\begin{aligned}
& +2\left(h_{t}^{2}-\frac{1}{2} g_{s}^{2}\right)^{2} D_{S S S}\left(m_{\omega}, m_{\tilde{t}_{L 1}}, m_{\tilde{t}_{L 2}}\right)+2\left(\frac{g_{s}^{2}}{6}\right)^{2} D_{S S S}\left(m_{u}, m_{\tilde{t}_{L 2}}, m_{\tilde{t}_{L 2}}\right) \\
& +\left(h_{t}^{2}-\frac{1}{3} g_{s}^{2}\right)^{2} D_{S S S}\left(m_{u}, m_{\tilde{b}_{L 1}}, m_{\tilde{b}_{L 1}}\right) \\
& +2\left(h_{t}^{2}-\frac{1}{2} g_{s}^{2}\right)^{2} D_{S S S}\left(m_{\omega}, m_{\tilde{b}_{L 1}}, m_{\tilde{b}_{L 2}}\right)+2\left(\frac{g_{s}^{2}}{6}\right)^{2} D_{S S S}\left(m_{u}, m_{\tilde{b}_{L 2}}, m_{\tilde{b}_{L 2}}\right) \\
& +\left(\frac{1}{3} g_{s}^{2}\right)^{2} D_{S S S}\left(m_{u}, m_{\tilde{b}_{R 1}}, m_{\tilde{b}_{R 1}}\right)+2\left(\frac{1}{2} g_{s}^{2}\right)^{2} D_{S S S}\left(m_{\omega}, m_{\tilde{b}_{R 1}}, m_{\tilde{b}_{R 2}}\right) \\
& \left.+2\left(\frac{g_{s}^{2}}{6}\right)^{2} D_{S S S}\left(m_{u}, m_{\tilde{b}_{R 2}}, m_{\tilde{b}_{R 2}}\right)\right]
\end{aligned}
$$

$$
\begin{aligned}
& (S S)=\frac{1}{4}\left(\frac{g_{w}^{2}}{8}+\frac{g_{s}^{2}}{6}\right)\left[8 D_{S S}\left(m_{\tilde{t}_{L 1}}, m_{\tilde{t}_{L 1}}\right)+24 D_{S S}\left(m_{\tilde{t}_{L 2}}, m_{\tilde{t}_{L 2}}\right)+16 D_{S S}\left(m_{\tilde{t}_{L 1}}, m_{\tilde{t}_{L 2}}\right)\right. \\
& \left.+8 D_{S S}\left(m_{\tilde{b}_{L 1}}, m_{\tilde{b}_{L 1}}\right)+24 D_{S S}\left(m_{\tilde{b}_{L 2}}, m_{\tilde{b}_{L 2}}\right)+16 D_{S S}\left(m_{\tilde{b}_{L 1}}, m_{\tilde{b}_{L 2}}\right)\right] \\
& +\frac{1}{4}\left(\frac{g_{s}^{2}}{6}\right)\left[8 D_{S S}\left(m_{\tilde{t}_{R 1}}, m_{\tilde{t}_{R 1}}\right)+24 D_{S S}\left(m_{\tilde{t}_{R 2}}, m_{\tilde{t}_{R 2}}\right)+16 D_{S S}\left(m_{\tilde{t}_{R 1}}, m_{\tilde{t}_{R 2}}\right)\right. \\
& \left.+8 D_{S S}\left(m_{\tilde{b}_{R 1}}, m_{\tilde{b}_{R 1}}\right)+24 D_{S S}\left(m_{\tilde{b}_{R 2}}, m_{\tilde{b}_{R 2}}\right)+16 D_{S S}\left(m_{\tilde{b}_{R 1}}, m_{\tilde{b}_{R 2}}\right)\right] \\
& +h_{t}^{2}\left[D_{S S}\left(m_{\tilde{t}_{L 1}}, m_{\tilde{t}_{R 1}}\right)+D_{S S}\left(m_{\tilde{t}_{L 2}}, m_{\tilde{t}_{R 2}}\right)+D_{S S}\left(m_{\tilde{t}_{L 3}}, m_{\tilde{t}_{R 3}}\right)\right] \\
& +h_{t}^{2}\left[D_{S S}\left(m_{\tilde{b}_{L 1}}, m_{\tilde{t}_{R 1}}\right)+D_{S S}\left(m_{\tilde{b}_{L 2}}, m_{\tilde{t}_{R 2}}\right)+D_{S S}\left(m_{\tilde{b}_{L 3}}, m_{\tilde{t}_{R 3}}\right)\right] \\
& +\left(\frac{1}{2} h_{t}^{2}+\frac{1}{8} g_{w}^{2} \cos 2 \beta\right)\left[D_{S S}\left(m_{\tilde{t}_{L 1}}, m_{h}\right)+D_{S S}\left(m_{\tilde{t}_{L 2}}, m_{h}\right)+D_{S S}\left(m_{\tilde{t}_{L 3}}, m_{h}\right)\right. \\
& +2\left(D_{S S}\left(m_{\tilde{b}_{L 1}}, m_{\pi}\right)+D_{S S}\left(m_{\tilde{b}_{L 2}}, m_{\pi}\right)+D_{S S}\left(m_{\tilde{b}_{L 3}}, m_{\pi}\right)\right) \\
& \left.+D_{S S}\left(m_{\tilde{t}_{L 1}}, m_{\pi}\right)+D_{S S}\left(m_{\tilde{t}_{L 2}}, m_{\pi}\right)+D_{S S}\left(m_{\tilde{t}_{L 3}}, m_{\pi}\right)\right] \\
& -\left(\frac{1}{8} g_{w}^{2} \cos 2 \beta\right)\left[D_{S S}\left(m_{\tilde{b}_{L 1}}, m_{h}\right)+D_{S S}\left(m_{\tilde{b}_{L 2}}, m_{h}\right)+D_{S S}\left(m_{\tilde{b}_{L 3}}, m_{h}\right)\right. \\
& +2\left(D_{S S}\left(m_{\tilde{t}_{L 1}}, m_{\pi}\right)+D_{S S}\left(m_{\tilde{t}_{L 2}}, m_{\pi}\right)+D_{S S}\left(m_{\tilde{t}_{L 3}}, m_{\pi}\right)\right) \\
& \left.+D_{S S}\left(m_{\tilde{b}_{L 1}}, m_{\pi}\right)+D_{S S}\left(m_{\tilde{b}_{L 2}}, m_{\pi}\right)+D_{S S}\left(m_{\tilde{b}_{L 3}}, m_{\pi}\right)\right] \\
& +\frac{1}{2} h_{t}^{2} \sin ^{2} \beta\left[D_{S S}\left(m_{\tilde{t}_{R 1}}, m_{h}\right)+D_{S S}\left(m_{\tilde{t}_{R 2}}, m_{h}\right)+D_{S S}\left(m_{\tilde{t}_{R 3}}, m_{h}\right)\right. \\
& \left.+3\left(D_{S S}\left(m_{\tilde{t}_{R 1}}, m_{\pi}\right)+D_{S S}\left(m_{\tilde{t}_{R 2}}, m_{\pi}\right)+D_{S S}\left(m_{\tilde{t}_{R 3}}, m_{\pi}\right)\right)\right]+\frac{g_{w}^{2}}{4}\left(2-N_{c}\right)\left[D_{S S}\left(m_{\tilde{t}_{L 1}}, m_{\tilde{b}_{L 1}}\right)\right. \\
& \left.+D_{S S}\left(m_{\tilde{t}_{L 2}}, m_{\tilde{b}_{L 2}}\right)+D_{S S}\left(m_{\tilde{t}_{L 3}}, m_{\tilde{b}_{L 3}}\right)\right] \text {. }
\end{aligned}
$$


The counterterm contribution to the mass term comes from an analogous contribution to that of eq. (49) and is

$$
\delta V=\frac{T^{2}}{16 \pi^{2}} \frac{\chi^{2}}{2} \frac{146}{27} g_{s}^{4}
$$

\subsection{Integration over the heavy scale}

The second part of the calculation arises, as noticed in the paper by Kajantie et al. [21]: when the "heavy" particles have been integrated out their contributions to the 3D mass parameters should also be included, as they can substantially vary the value of the parameters $\Lambda_{H_{3}}, \Lambda_{U_{3}}$. In order to do this we must calculate the 2-loop contributions to the effective potential in the $\phi$ - and $\chi$-directions from the heavy fields: $Q, D, C_{o}, A_{o}$

For the $\phi$-direction the expression of the effective potential to 2-loops from the heavy particles can be deduced from the expressions in the paper by Espinosa [12]. For the $\chi$-direction this is new. The masses in the shifted theory are now given by

$$
\begin{gathered}
m_{\tilde{t}_{L_{1}}}^{2}=\bar{m}_{Q_{3}}^{2}+\left(h_{t}^{L}+\Lambda_{3}+\Lambda_{4}^{s}\right) \frac{\phi^{2}}{2}+\left(h_{t}^{Q U}+g_{s_{1}}^{Q U}+g_{s_{2}}^{Q U}\right) \frac{\chi^{2}}{2} \\
m_{\tilde{t}_{L_{2}, 3}}^{2}=\bar{m}_{Q_{3}}^{2}+\left(h_{t}^{L}+\Lambda_{3}+\Lambda_{4}^{s}\right) \frac{\phi^{2}}{2}+\left(g_{s_{2}}^{Q U}\right) \frac{\chi^{2}}{2} \\
m_{\tilde{b}_{L_{1}}}^{2}=\bar{m}_{Q_{3}}^{2}+\left(\Lambda_{3}+\Lambda_{4}^{c}\right) \frac{\phi^{2}}{2}+\left(h_{t}^{Q U}+g_{s_{1}}^{Q U}+g_{s_{2}}^{Q U}\right) \frac{\chi^{2}}{2} \\
m_{\tilde{b}_{L_{2,3}}}^{2}=\bar{m}_{Q_{3}}^{2}+\left(\Lambda_{3}+\Lambda_{4}^{c}\right) \frac{\phi^{2}}{2}+\left(g_{s_{2}}^{Q U}\right) \frac{\chi^{2}}{2} \\
m_{\tilde{b}_{R_{1}}}^{2}=m_{D_{3}}^{2}+\left(g_{s_{1}}^{U D}+g_{s_{2}}^{U D}\right) \frac{\chi^{2}}{2} \\
m_{\tilde{b}_{R_{2,3}}}^{2}=\bar{m}_{D_{3}}^{2}+\left(g_{s_{2}}^{Q U}\right) \frac{\chi^{2}}{2} .
\end{gathered}
$$

\footnotetext{
${ }^{9}$ There is an additional counterterm contribution in the $\chi$-direction for the case of a light higgsino.
} 
The expressions for the rest of the fields are given in [14. The 2-loop contributions from the heavy scale are given below. We stress that the $D$-integrals in eqs. (65) and (66) are just $3 D$ integrals, our notation follows that of refs. [14, 20, 21] $]^{10}$.

\subsection{1 $\phi$-direction}

$$
\begin{aligned}
& V_{2}^{\text {heavy }}=\frac{3}{8} g_{w_{3}}^{2}\left[D_{L S}\left(m_{h}, m_{A_{0}}\right)+3 D_{L S}\left(m_{\pi}, m_{A_{0}}\right)\right]+3 g_{w_{3}}^{2} D_{L V}\left(m_{W}, m_{A_{0}}\right) \\
& \text { - } \frac{3}{16} g_{w_{3}}^{2} \phi^{2} D_{L L S}\left(m_{h}, m_{A_{0}}, m_{A_{0}}\right)-\frac{3}{2} g_{w_{3}}^{2} D_{L L V}\left(m_{W}, m_{A_{0}}, m_{A_{0}}\right) \\
& -\frac{g_{w_{3}}^{2}}{8} N_{c}\left[D_{S S V}\left(m_{\tilde{t}_{L}}, m_{\tilde{t}_{L}}, m_{W}\right)+D_{S S V}\left(m_{\tilde{b}_{L}}, m_{\tilde{b}_{L}}, m_{W}\right)+4 D_{S S V}\left(m_{\tilde{t}_{L}}, m_{\tilde{b}_{L}}, m_{W}\right)\right] \\
& -\frac{g_{s_{3}}^{2}}{4}\left(N_{c}^{2}-1\right)\left[D_{S S V}\left(m_{\tilde{t}_{L}}, m_{\tilde{t}_{L}}, 0\right)+D_{S S V}\left(m_{\tilde{b}_{L}}, m_{\tilde{b}_{L}}, 0\right)+D_{S S V}\left(m_{\tilde{b}_{R}}, m_{\tilde{b}_{R}}, 0\right)\right] \\
& -\left[\left(h_{t}^{L}+\Lambda_{3}+\Lambda_{4}^{s}\right)^{2} D_{S S S}\left(m_{\tilde{t}_{L}}, m_{\tilde{t}_{L}}, m_{h}\right)+\left(\Lambda_{3}+\Lambda_{4}^{c}\right)^{2} D_{S S S}\left(m_{\tilde{b}_{L}}, m_{\tilde{b}_{L}}, m_{h}\right)\right. \\
& \left.+\left(h_{t}^{L}-\Lambda_{4}^{c}+\Lambda_{4}^{s}\right)^{2} D_{S S S}\left(m_{\tilde{t}_{L}}, m_{\tilde{b}_{L}}, m_{\pi}\right)\right] \frac{\phi^{2}}{2} N_{c} \\
& -\frac{1}{4} g_{s_{3}}^{2}\left(N_{c}^{2}-1\right)\left[D_{S V}\left(m_{\tilde{t}_{L}}, 0\right)+D_{S V}\left(m_{\tilde{b}_{L}}, 0\right)+D_{S V}\left(m_{\tilde{b}_{R}}, 0\right)\right] \\
& -\frac{3}{8} g_{w_{3}}^{2} N_{c}\left[D_{S V}\left(m_{\tilde{t}_{L}}, m_{W}\right)+D_{S V}\left(m_{\tilde{b}_{L}}, m_{W}\right)\right] \\
& +\left(2 \Lambda_{1}+g_{s_{1}}^{Q Q}+g_{s_{2}}^{Q Q}\right) N_{c}\left(2-N_{c}\right) D_{S S}\left(m_{\tilde{t}_{L}}, m_{\tilde{b}_{L}}\right) \\
& +\left(h_{t}^{Q U}+g_{s_{1}}^{Q U}+3 g_{s_{2}}^{Q U}\right) N_{c}\left[D_{S S}\left(m_{\tilde{t}_{L}}, m_{\tilde{t}_{R}}\right)+D_{S S}\left(m_{\tilde{b}_{L}}, m_{\tilde{t}_{R}}\right)\right] \\
& +\left(\Lambda_{1}+\lambda_{Q_{3}}\right) N_{c}\left(N_{c}+1\right)\left[D_{S S}\left(m_{\tilde{t}_{L}}, m_{\tilde{t}_{L}}\right)+D_{S S}\left(m_{\tilde{b}_{L}}, m_{\tilde{b}_{L}}\right)\right] \\
& +\lambda_{D_{3}} N_{c}\left(N_{c}+1\right)\left[D_{S S}\left(m_{\tilde{b}_{R}}, m_{\tilde{b}_{R}}\right)\right] \\
& +N_{c} \frac{1}{2}\left(h_{t}^{L}+\Lambda_{3}+\Lambda_{4}^{s}\right)\left[D_{S S}\left(m_{\tilde{t}_{L}}, m_{h}\right)+2 D_{S S}\left(m_{\tilde{b}_{L}}, m_{\pi}\right)+D_{S S}\left(m_{\tilde{t}_{L}}, m_{\pi}\right)\right] \\
& +\frac{1}{2} N_{c}\left(\Lambda_{3}+\Lambda_{4}^{c}\right)\left[D_{S S}\left(m_{\tilde{b}_{L}}, m_{h}\right)+2 D_{S S}\left(m_{\tilde{t}_{L}}, m_{\pi}\right)+D_{S S}\left(m_{\tilde{b}_{L}}, m_{\pi}\right)\right] \\
& +\left(g_{s_{1}}^{Q D}+3 g_{s_{2}}^{Q D}\right) N_{c}\left[D_{S S}\left(m_{\tilde{t}_{L}}, m_{\tilde{b}_{R}}\right)+D_{S S}\left(m_{\tilde{b}_{L}}, m_{\tilde{b}_{R}}\right)\right] \\
& +\left(g_{s_{1}}^{U D}+3 g_{s_{2}}^{U D}\right) N_{c}\left[D_{S S}\left(m_{\tilde{t}_{R}}, m_{\tilde{b}_{R}}\right)\right] \text {. }
\end{aligned}
$$

\footnotetext{
${ }^{10}$ Our convention for the functions $D_{V V V}, D_{V V S}$ is that of [14].
} 


\subsection{2 $\chi$-direction}

$$
\begin{aligned}
& V_{2}^{\text {heavy }}=\frac{g_{s_{3}}^{2}}{4}\left(\left(N_{c}-1\right)\left[D_{L S}\left(m_{u}, m_{C_{0}}\right)+D_{L S}\left(m_{\omega}, m_{C_{0}}\right)\right]\right. \\
& \left.+\frac{1}{N_{c}}\left[4 D_{L S}\left(m_{\omega}, m_{C_{0}}\right)+2 D_{L S}\left(m_{u}, m_{C_{0}}\right)\right]\right) \\
& -g_{s_{3}}^{2} \frac{N_{c}}{2}\left[\left(N_{c}-1\right) D_{L L S}\left(m_{C_{0}}, m_{C_{0}}, m_{u}\right)+2 \frac{\left(N_{c}-1\right)^{2}}{N_{c}^{2}} D_{L L S}\left(m_{C_{0}}, m_{C_{0}}, m_{u}\right)\right. \\
& \left.+N_{c}\left(N_{c}-2\right) D_{L L S}\left(0, m_{C_{0}}, m_{\omega}\right)+\frac{\left(N_{c}-2\right)^{2}}{N_{c}} D_{L L S}\left(m_{C_{0}}, m_{C_{0}}, m_{\omega}\right)\right] \\
& -g_{s_{3}}^{2} \frac{N_{c}}{2}\left[-D_{L V}\left(m_{C_{0}}, \bar{m}_{G}\right)-D_{L V}\left(m_{C_{0}}, m_{G}\right)-\left(N_{c}-1\right) D_{L V}\left(m_{C_{0}}, m_{G}\right)\right] \\
& \text { - } g_{s_{3}}^{2} \frac{N_{c}}{4}\left[D_{L L V}\left(m_{C_{0}}, m_{C_{0}}, \bar{m}_{G}\right)+2 D_{L L V}\left(m_{C_{0}}, m_{C_{0}}, m_{G}\right)\right. \\
& \left.+D_{L L V}\left(m_{C_{0}}, m_{C_{0}}, 0\right)+2 D_{L L V}\left(m_{C_{0}}, 0, m_{G}\right)\right] \\
& -\frac{g_{w_{3}}^{2}}{8}\left[D_{S S V}\left(m_{\tilde{t}_{L 1}}, m_{\tilde{t}_{L 1}}, 0\right)+D_{S S V}\left(m_{\tilde{t}_{L 2}}, m_{\tilde{t}_{L 2}}, 0\right)+D_{S S V}\left(m_{\tilde{t}_{L 3}}, m_{\tilde{t}_{L 3}}, 0\right)\right. \\
& +D_{S S V}\left(m_{\tilde{b}_{L 1}}, m_{\tilde{b}_{L 1}}, 0\right)+D_{S S V}\left(m_{\tilde{b}_{L 2}}, m_{\tilde{b}_{L 2}}, 0\right)+D_{S S V}\left(m_{\tilde{b}_{L 3}}, m_{\tilde{b}_{L 3}}, 0\right) \\
& \left.+4\left(D_{S S V}\left(m_{\tilde{t}_{L 1}}, m_{\tilde{b}_{L 1}}, 0\right)+D_{S S V}\left(m_{\tilde{t}_{L 2}}, m_{\tilde{b}_{L 2}}, 0\right)+D_{S S V}\left(m_{\tilde{t}_{L 3}}, m_{\tilde{b}_{L 3}}, 0\right)\right)\right] \\
& -g_{s_{3}}^{2} \frac{1}{4}\left[+2\left(N_{c}-1\right) D_{S S V}\left(m_{\tilde{t}_{L 1}}, m_{\tilde{t}_{L 2}}, m_{G}\right)+\frac{N_{c}-1}{N_{c}} D_{S S V}\left(m_{\tilde{t}_{L 1}}, m_{\tilde{t}_{L 1}}, \bar{m}_{G}\right)\right. \\
& +\frac{1}{N_{c}} D_{S S V}\left(m_{\tilde{t}_{L 2}}, m_{\tilde{t}_{L 2}}, \bar{m}_{G}\right)+N_{c}\left(N_{c}-2\right) D_{S S V}\left(m_{\tilde{t}_{L 2}}, m_{\tilde{t}_{L 2}}, 0\right) \\
& +2\left(N_{c}-1\right) D_{S S V}\left(m_{\tilde{b}_{L 1}}, m_{\tilde{b}_{L 2}}, m_{G}\right)+\frac{N_{c}-1}{N_{c}} D_{S S V}\left(m_{\tilde{b}_{L 1}}, m_{\tilde{b}_{L 1}}, \bar{m}_{G}\right) \\
& +\frac{1}{N_{c}} D_{S S V}\left(m_{\tilde{b}_{L 2}}, m_{\tilde{b}_{L 2}}, \bar{m}_{G}\right) \\
& +N_{c}\left(N_{c}-2\right) D_{S S V}\left(m_{\tilde{b}_{L 2}}, m_{\tilde{b}_{L 2}}, 0\right)+2\left(N_{c}-1\right) D_{S S V}\left(m_{\tilde{b}_{R 1}}, m_{\tilde{b}_{R 2}}, m_{G}\right) \\
& +\frac{N_{c}-1}{N_{c}} D_{S S V}\left(m_{\tilde{b}_{R 1}}, m_{\tilde{b}_{R 1}}, \bar{m}_{G}\right) \\
& \left.+\frac{1}{N_{c}} D_{S S V}\left(m_{\tilde{b}_{R 2}}, m_{\tilde{b}_{R 2}}, \bar{m}_{G}\right)+N_{c}\left(N_{c}-2\right) D_{S S V}\left(m_{\tilde{b}_{R 2}}, m_{\tilde{b}_{R 2}}, 0\right)\right] \\
& -\frac{\chi^{2}}{2}\left[\left(h_{t}^{Q U}+g_{s_{1}}^{Q U}+g_{s_{2}}^{Q U}\right)^{2} D_{S S S}\left(m_{u}, m_{\tilde{t}_{L 1}}, m_{\tilde{t}_{L 1}}\right)+2\left(h_{t}^{Q U}+g_{s_{1}}^{Q U}\right)^{2} D_{S S S}\left(m_{\omega}, m_{\tilde{t}_{L 1}}, m_{\tilde{t}_{L 2}}\right)\right.
\end{aligned}
$$




$$
\begin{aligned}
& +2\left(g_{s_{2}}^{Q U}\right)^{2} D_{S S S}\left(m_{u}, m_{\tilde{t}_{L 2}}, m_{\tilde{t}_{L 2}}\right)+\left(h_{t}^{Q U}+g_{s_{1}}^{Q U}+g_{s_{2}}^{Q U}\right)^{2} D_{S S S}\left(m_{u}, m_{\tilde{b}_{L 1}}, m_{\tilde{b}_{L 1}}\right) \\
& +2\left(h_{t}^{Q U}+g_{s_{1}}^{Q U}\right)^{2} D_{S S S}\left(m_{\omega}, m_{\tilde{b}_{L 1}}, m_{\tilde{b}_{L 2}}\right)+2\left(g_{s_{2}}^{Q U}\right)^{2} D_{S S S}\left(m_{u}, m_{\tilde{b}_{L 2}}, m_{\tilde{b}_{L 2}}\right) \\
& +\left(g_{s_{1}}^{U D}+g_{s_{2}}^{U D}\right)^{2} D_{S S S}\left(m_{u}, m_{\tilde{b}_{R 1}}, m_{\tilde{b}_{R 1}}\right)+2\left(g_{s_{1}}^{U D}\right)^{2} D_{S S S}\left(m_{\omega}, m_{\tilde{b}_{R 1}}, m_{\tilde{b}_{R 2}}\right) \\
& \left.+2\left(g_{s_{2}}^{U D}\right)^{2} D_{S S S}\left(m_{u}, m_{\tilde{b}_{R 2}}, m_{\tilde{b}_{R 2}}\right)\right] \\
& -\frac{g_{s 3}^{2}}{8}\left[2 N_{c}\left(N_{c}-2\right) D_{S V}\left(m_{\tilde{t}_{L 2}}, 0\right)+\left(N_{c}-1\right)\left[2 D_{S V}\left(m_{\tilde{t}_{L 2}}, m_{G}\right)+2 D_{S V}\left(m_{\tilde{t}_{L 1}}, m_{G}\right)\right]\right. \\
& +\frac{1}{N_{c}}\left[2 D_{S V}\left(m_{\tilde{t}_{L 2}}, \bar{m}_{G}\right)+2 D_{S V}\left(m_{\tilde{t}_{L 1}}, \bar{m}_{G}\right)\right] \\
& +2 N_{c}\left(N_{c}-2\right) D_{S V}\left(m_{\tilde{b}_{L 2}}, 0\right)+\left(N_{c}-1\right)\left[2 D_{S V}\left(m_{\tilde{b}_{L 2}}, m_{G}\right)+2 D_{S V}\left(m_{\tilde{b}_{L 1}}, m_{G}\right)\right] \\
& +\frac{1}{N_{c}}\left[2 D_{S V}\left(m_{\tilde{b}_{L 2}}, \bar{m}_{G}\right)+2 D_{S V}\left(m_{\tilde{b}_{L 1}}, \bar{m}_{G}\right)\right] \\
& +2 N_{c}\left(N_{c}-2\right) D_{S V}\left(m_{\tilde{b}_{R 2}}, 0\right)+\left(N_{c}-1\right)\left[2 D_{S V}\left(m_{\tilde{b}_{R 2}}, m_{G}\right)+2 D_{S V}\left(m_{\tilde{b}_{R 1}}, m_{G}\right)\right] \\
& \left.+\frac{1}{N_{c}}\left[2 D_{S V}\left(m_{\tilde{b}_{R 2}}, \bar{m}_{G}\right)+2 D_{S V}\left(m_{\tilde{b}_{R 1}}, \bar{m}_{G}\right)\right]\right] \\
& -\frac{3}{8} g_{w_{3}}^{2}\left[D_{S V}\left(m_{\tilde{t}_{L 1}}, 0\right)+D_{S V}\left(m_{\tilde{t}_{L 2}}, 0\right)+D_{S V}\left(m_{\tilde{t}_{L 3}}, 0\right)\right. \\
& \left.+D_{S V}\left(m_{\tilde{b}_{L 1}}, 0\right)+D_{S V}\left(m_{\tilde{b}_{L 2}}, 0\right)+D_{S V}\left(m_{\tilde{b}_{L 3}}, 0\right)\right] \\
& +\frac{1}{4}\left(\Lambda_{1}+\lambda_{Q_{3}}\right)\left[8 D_{S S}\left(m_{\tilde{t}_{L 1}}, m_{\tilde{t}_{L 1}}\right)+24 D_{S S}\left(m_{\tilde{t}_{L 2}}, m_{\tilde{t}_{L 2}}\right)+16 D_{S S}\left(m_{\tilde{t}_{L 1}}, m_{\tilde{t}_{L 2}}\right)\right. \\
& \left.+8 D_{S S}\left(m_{\tilde{b}_{L 1}}, m_{\tilde{b}_{L 1}}\right)+24 D_{S S}\left(m_{\tilde{b}_{L 2}}, m_{\tilde{b}_{L 2}}\right)+16 D_{S S}\left(m_{\tilde{b}_{L 1}}, m_{\tilde{b}_{L 2}}\right)\right] \\
& +\frac{1}{4}\left(\lambda_{D_{3}}\right)\left[8 D_{S S}\left(m_{\tilde{b}_{R 1}}, m_{\tilde{b}_{R 1}}\right)+24 D_{S S}\left(m_{\tilde{b}_{R 2}}, m_{\tilde{b}_{R 2}}\right)+16 D_{S S}\left(m_{\tilde{b}_{R 1}}, m_{\tilde{b}_{R 2}}\right)\right] \\
& +\left(h_{t}^{Q U}+g_{s_{1}}^{Q U}\right)\left[D_{S S}\left(m_{\tilde{t}_{L 1}}, m_{\tilde{t}_{R 1}}\right)+D_{S S}\left(m_{\tilde{t}_{L 2}}, m_{\tilde{t}_{R 2}}\right)+D_{S S}\left(m_{\tilde{t}_{L 3}}, m_{\tilde{t}_{R 3}}\right)\right] \\
& +\left(h_{t}^{Q U}+g_{s_{1}}^{Q U}\right)\left[D_{S S}\left(m_{\tilde{b}_{L 1}}, m_{\tilde{t}_{R 1}}\right)+D_{S S}\left(m_{\tilde{b}_{L 2}}, m_{\tilde{t}_{R 2}}\right)+D_{S S}\left(m_{\tilde{b}_{L 3}}, m_{\tilde{t}_{R 3}}\right)\right] \\
& +g_{s 2}^{Q U}\left[D_{S S}\left(m_{\tilde{t}_{L 1}}, m_{\tilde{t}_{R 1}}\right)+D_{S S}\left(m_{\tilde{t}_{L 1}}, m_{\tilde{t}_{R 2}}\right)+D_{S S}\left(m_{\tilde{t}_{L 1}}, m_{\tilde{t}_{R 3}}\right)\right. \\
& +D_{S S}\left(m_{\tilde{t}_{L 2}}, m_{\tilde{t}_{R 1}}\right)+D_{S S}\left(m_{\tilde{t}_{L 2}}, m_{\tilde{t}_{R 2}}\right)+D_{S S}\left(m_{\tilde{t}_{L 2}}, m_{\tilde{t}_{R 3}}\right) \\
& +D_{S S}\left(m_{\tilde{t}_{L 3}}, m_{\tilde{t}_{R 1}}\right)+D_{S S}\left(m_{\tilde{t}_{L 3}}, m_{\tilde{t}_{R 2}}\right)+D_{S S}\left(m_{\tilde{t}_{L 3}}, m_{\tilde{t}_{R 3}}\right) \\
& +D_{S S}\left(m_{\tilde{b}_{L 1}}, m_{\tilde{t}_{R 1}}\right)+D_{S S}\left(m_{\tilde{b}_{L 1}}, m_{\tilde{t}_{R 2}}\right)+D_{S S}\left(m_{\tilde{b}_{L 1}}, m_{\tilde{t}_{R 3}}\right) \\
& +D_{S S}\left(m_{\tilde{b}_{L 2}}, m_{\tilde{t}_{R 1}}\right)+D_{S S}\left(m_{\tilde{b}_{L 2}}, m_{\tilde{t}_{R 2}}\right)+D_{S S}\left(m_{\tilde{b}_{L 2}}, m_{\tilde{t}_{R 3}}\right)+D_{S S}\left(m_{\tilde{b}_{L 3}}, m_{\tilde{t}_{R 1}}\right) \\
& \left.+D_{S S}\left(m_{\tilde{b}_{L 3}}, m_{\tilde{t}_{R 2}}\right)+D_{S S}\left(m_{\tilde{b}_{L 3}}, m_{\tilde{t}_{R 3}}\right)\right]
\end{aligned}
$$




$$
\begin{aligned}
& +\frac{1}{2}\left(h_{t}^{L}+\Lambda_{3}+\Lambda_{4}^{s}\right)\left[D_{S S}\left(m_{\tilde{t}_{L 1}}, m_{h}\right)+D_{S S}\left(m_{\tilde{t}_{L 2}}, m_{h}\right)+D_{S S}\left(m_{\tilde{t}_{L 3}}, m_{h}\right)\right. \\
& +2\left(D_{S S}\left(m_{\tilde{b}_{L 1}}, m_{\pi}\right)+D_{S S}\left(m_{\tilde{b}_{L 2}}, m_{\pi}\right)+D_{S S}\left(m_{\tilde{b}_{L 3}}, m_{\pi}\right)\right) \\
& \left.+D_{S S}\left(m_{\tilde{t}_{L 1}}, m_{\pi}\right)+D_{S S}\left(m_{\tilde{t}_{L 2}}, m_{\pi}\right)+D_{S S}\left(m_{\tilde{t}_{L 3}}, m_{\pi}\right)\right] \\
& +\left(\Lambda_{3}+\Lambda_{4}^{c}\right)\left[D_{S S}\left(m_{\tilde{b}_{L 1}}, m_{h}\right)+D_{S S}\left(m_{\tilde{b}_{L 2}}, m_{h}\right)+D_{S S}\left(m_{\tilde{b}_{L 3}}, m_{h}\right)\right. \\
& +2\left(D_{S S}\left(m_{\tilde{t}_{L 1}}, m_{\pi}\right)+D_{S S}\left(m_{\tilde{t}_{L 2}}, m_{\pi}\right)+D_{S S}\left(m_{\tilde{t}_{L 3}}, m_{\pi}\right)\right) \\
& \left.+D_{S S}\left(m_{\tilde{b}_{L 1}}, m_{\pi}\right)+D_{S S}\left(m_{\tilde{b}_{L 2}}, m_{\pi}\right)+D_{S S}\left(m_{\tilde{b}_{L 3}}, m_{\pi}\right)\right] \\
& +2 \Lambda_{1}\left(2-N_{c}\right)\left[D_{S S}\left(m_{\tilde{t}_{L 1}}, m_{\tilde{b}_{L 1}}\right)+D_{S S}\left(m_{\tilde{t}_{L 2}}, m_{\tilde{b}_{L 2}}\right)+D_{S S}\left(m_{\tilde{t}_{L 3}}, m_{\tilde{b}_{L 3}}\right)\right] \\
& +g_{s 1}^{Q D}\left[D_{S S}\left(m_{\tilde{t}_{L 1}}, m_{\tilde{b}_{R 1}}\right)+D_{S S}\left(m_{\tilde{t}_{L 2}}, m_{\tilde{b}_{R 2}}\right)+D_{S S}\left(m_{\tilde{t}_{L 3}}, m_{\tilde{b}_{R 3}}\right)\right] \\
& +g_{s_{1}}^{Q D}\left[D_{S S}\left(m_{\tilde{b}_{L 1}}, m_{\tilde{b}_{R 1}}\right)+D_{S S}\left(m_{\tilde{b}_{L 2}}, m_{\tilde{b}_{R 2}}\right)+D_{S S}\left(m_{\tilde{b}_{L 3}}, m_{\tilde{b}_{R 3}}\right)\right] \\
& +g_{s_{2}}^{Q D}\left[D_{S S}\left(m_{\tilde{t}_{L 1}}, m_{\tilde{b}_{R 1}}\right)+D_{S S}\left(m_{\tilde{t}_{L 1}}, m_{\tilde{b}_{R 2}}\right)+D_{S S}\left(m_{\tilde{t}_{L 1}}, m_{\tilde{b}_{R 3}}\right)\right. \\
& +D_{S S}\left(m_{\tilde{t}_{L 2}}, m_{\tilde{b}_{R 1}}\right)+D_{S S}\left(m_{\tilde{t}_{L 2}}, m_{\tilde{b}_{R 2}}\right)+D_{S S}\left(m_{\tilde{t}_{L 2}}, m_{\tilde{b}_{R 3}}\right) \\
& +D_{S S}\left(m_{\tilde{t}_{L 3}}, m_{\tilde{b}_{R 1}}\right)+D_{S S}\left(m_{\tilde{t}_{L 3}}, m_{\tilde{b}_{R 2}}\right) \\
& +D_{S S}\left(m_{\tilde{t}_{L 3}}, m_{\tilde{b}_{R 3}}\right)+D_{S S}\left(m_{\tilde{b}_{L 1}}, m_{\tilde{b}_{R 1}}\right)+D_{S S}\left(m_{\tilde{b}_{L 1}}, m_{\tilde{b}_{R 2}}\right)+D_{S S}\left(m_{\tilde{b}_{L 1}}, m_{\tilde{b}_{R 3}}\right) \\
& +D_{S S}\left(m_{\tilde{b}_{L 2}}, m_{\tilde{b}_{R 1}}\right)+D_{S S}\left(m_{\tilde{b}_{L 2}}, m_{\tilde{b}_{R 2}}\right)+D_{S S}\left(m_{\tilde{b}_{L 2}}, m_{\tilde{b}_{R 3}}\right)+D_{S S}\left(m_{\tilde{b}_{L 3}}, m_{\tilde{b}_{R 1}}\right) \\
& \left.+D_{S S}\left(m_{\tilde{b}_{L 3}}, m_{\tilde{b}_{R 2}}\right)+D_{S S}\left(m_{\tilde{b}_{L 3}}, m_{\tilde{b}_{R 3}}\right)\right] \\
& +g_{s_{1}}^{U D}\left[D_{S S}\left(m_{\tilde{t}_{R 1}}, m_{\tilde{b}_{R 1}}\right)+D_{S S}\left(m_{\tilde{t}_{R 2}}, m_{\tilde{b}_{R 2}}\right)+D_{S S}\left(m_{\tilde{t}_{R 3}}, m_{\tilde{b}_{R 3}}\right)\right] \\
& +g_{s_{2}}^{U D}\left[D_{S S}\left(m_{\tilde{t}_{R 1}}, m_{\tilde{b}_{R 1}}\right)+D_{S S}\left(m_{\tilde{t}_{R 1}}, m_{\tilde{b}_{R 2}}\right)+D_{S S}\left(m_{\tilde{t}_{R 1}}, m_{\tilde{b}_{R 3}}\right)\right. \\
& +D_{S S}\left(m_{\tilde{t}_{R 2}}, m_{\tilde{b}_{R 1}}\right)+D_{S S}\left(m_{\tilde{t}_{R 2}}, m_{\tilde{b}_{R 2}}\right)+D_{S S}\left(m_{\tilde{t}_{R 2}}, m_{\tilde{b}_{R 3}}\right) \\
& +D_{S S}\left(m_{\tilde{t}_{R 3}}, m_{\tilde{b}_{R 1}}\right)+D_{S S}\left(m_{\tilde{t}_{R 3}}, m_{\tilde{b}_{R 2}}\right) \\
& \left.+D_{S S}\left(m_{\tilde{t}_{R 3}}, m_{\tilde{b}_{R 3}}\right)\right] \text {. }
\end{aligned}
$$

\subsubsection{Mass terms}

Using the results presented in the previous sections, we can finally write the full expressions for the mass terms of eqs. (35) and (36) 


$$
\begin{aligned}
& \bar{m}_{U_{3}}^{2}(\mu)=m_{U}^{2}\left(1+4 g_{s}^{2} \frac{L_{b}}{16 \pi^{2}}\right)+T\left(\frac{1}{3} g_{s_{3}}^{2}+\frac{2}{3} \lambda_{U_{3}}+\frac{1}{6} \gamma_{3}+\frac{1}{6}\left(h_{t}^{Q U}+g_{s_{1}}^{Q U}+3 g_{s_{2}}^{Q U}+g_{s_{1}}^{U D}+3 g_{s_{2}}^{U D}\right)\right) \\
& -\frac{L_{b}}{16 \pi^{2}}\left(\frac{4}{3} g_{s}^{2} m_{U}^{2}+2 h_{t}^{2} \sin ^{2} \beta\left(m_{H}^{2}+m_{Q}^{2}\right)\right) \\
& +\frac{1}{\left(16 \pi^{2}\right)}\left(8 \bar{g}_{s_{3}}^{4}+\frac{64}{3} \bar{\lambda}_{U_{3}} \bar{g}_{s_{3}}^{2}-16 \bar{\lambda}_{U_{3}}^{2}-2 \bar{\gamma}_{3}^{2}+3 \bar{g}_{w_{3}}^{2} \bar{\gamma}_{3}\right)\left(\log \left(\frac{3 T}{\mu}\right)+c\right) \\
& +\frac{T^{2}}{\left(16 \pi^{2}\right)}\left(g_{s}^{4}\left(\frac{146}{27}+\frac{2}{3}+\frac{11}{54}\right)\right) \\
& +\frac{1}{\left(16 \pi^{2}\right)}\left(-\frac{1}{4} g_{s_{3}}^{4}\left(\frac{29}{9}\left(\log \left(\frac{3 T}{\left(2 m_{C_{o}}\right)}\right)+c+\frac{1}{2}\right)+3\left(\log \left(\frac{3 T}{m_{C_{o}}}\right)+c+\frac{1}{2}\right)\right)\right. \\
& \left.+\frac{21}{4} g_{s_{3}}^{4}\left(\log \left(\frac{3 T}{\left(2 m_{C_{o}}\right)}\right)+c+\frac{1}{2}\right)\right) \\
& +\frac{1}{\left(16 \pi^{2}\right)}\left(\left(2\left(h_{t}^{Q U}+g_{s_{1}}^{Q U}+g_{s_{2}}^{Q U}\right)^{2}+8\left(h_{t}^{Q U}+g_{s_{1}}^{Q U}\right)^{2}+4\left(g_{s_{2}}^{Q U}\right)^{2}\right)\left(\log \left(\frac{3 T}{\left(2 m_{Q_{3}}\right)}\right)+c+\frac{1}{2}\right)\right. \\
& +\left(\left(g_{s_{1}}^{U D}+g_{s_{2}}^{U D}\right)^{2}+\left(g_{s_{2}}^{U D}\right)^{2}+4\left(g_{s_{1}}^{U D}\right)^{2}\right)\left(\log \left(\frac{3 T}{\left(2 m_{D_{3}}\right)}\right)+c+\frac{1}{2}\right) \\
& +\frac{1}{4} g_{s_{3}}^{2}\left(\left(8\left(g_{s_{3}}^{2}-2\left(h_{t}^{Q U}+g_{s_{1}}^{Q U}+g_{s_{2}}^{Q U}\right)-2 g_{s_{2}}^{Q U}\right)+\frac{4}{3}\left(\frac{2}{3} g_{s_{3}}^{2}-4\left(h_{t}^{Q U}+g_{s_{1}}^{Q U}+g_{s_{2}}^{Q U}\right)\right)\right.\right. \\
& \left.+\frac{2}{3}\left(\frac{2}{3} g_{s_{3}}^{2}-4 g_{s_{2}}^{Q U}\right)-24 g_{s_{2}}^{Q U}\right) \\
& \bar{m}_{H_{3}}^{2}(\mu)=m_{H}^{2}\left(1+\frac{9}{4} g_{w}^{2} \frac{L_{b}}{16 \pi^{2}}-3 h_{t}^{2} \frac{L_{f}}{16 \pi^{2}}\right)+T\left(\frac{1}{2} \lambda_{H_{3}}+\frac{3}{16} g_{w_{3}}^{2}+\frac{1}{16} g^{\prime 2} T\right. \\
& +\frac{1}{4} h_{t}^{f}+\frac{1}{4}\left(h_{t}^{L}+2 \Lambda_{3}^{Q}+\Lambda_{4}^{c}+\Lambda_{4}^{s}+\gamma_{3}\right) \\
& -\frac{L_{b}}{16 \pi^{2}}\left(6 \lambda m_{H}^{2}+3\left(m_{Q}^{2}+m_{U}^{2}\right) h_{t}^{2} \sin ^{2} \beta\right) \\
& +\frac{1}{\left(16 \pi^{2}\right)}\left(\frac{51}{16} \bar{g}_{w_{3}}^{4}+9 \bar{\lambda}_{H_{3}} \bar{g}_{w_{3}}^{2}-12 \bar{\lambda}_{H_{3}}^{2}-3 \bar{\gamma}_{3}^{2}+8 \bar{g}_{s_{3}}^{2} \bar{\gamma}_{3}\right)\left(\log \left(\frac{3 T}{\mu}\right)+c\right) \\
& +\frac{T^{2}}{\left(16 \pi^{2}\right)}\left(g_{w}^{4}\left(\frac{137}{96}+\frac{9}{2} \log 2+\frac{1}{4}\right)+\frac{3}{4} \lambda g_{w}^{2}\right) \\
& +\frac{1}{\left(16 \pi^{2}\right)}\left(\frac{15}{8} g_{w_{3}}^{4}\left(\log \left(\frac{3 T}{\left(2 m_{A_{o}}\right)}\right)+c\right)+\frac{9}{16} g_{w_{3}}^{4}\right)+\frac{T^{2}}{\left(16 \pi^{2}\right)}\left(\frac{2}{3} g_{s}^{2} h_{t}^{2} \sin ^{2} \beta\right)
\end{aligned}
$$




$$
\begin{aligned}
& +\frac{1}{\left(16 \pi^{2}\right)}\left(-\frac{3}{8} g_{w_{3}}^{2}\left(3 g_{w_{3}}^{2}-12\left(h_{t}^{L}+\Lambda_{3}^{Q}+\Lambda_{4}^{s}\right)-12\left(\Lambda_{3}^{Q}+\Lambda_{4}^{c}\right)\right)\left(\log \left(\frac{3 T}{\left(2 m_{Q_{3}}\right)}\right)+c+\frac{1}{2}\right)\right. \\
& -2 g_{s_{3}}^{2}\left(-4\left(h_{t}^{L}+\Lambda_{3}^{c}+\Lambda_{4}^{s}\right)-4\left(\Lambda_{3}^{Q}+\Lambda_{4}^{c}\right)\right)\left(\log \left(\frac{3 T}{\left(2 m_{Q_{3}}\right)}\right)+c+\frac{1}{2}\right) \\
& \left.-3\left(\left(h_{t}^{L}+\Lambda_{3}^{Q}+\Lambda_{4}^{s}\right)^{2}+\left(\Lambda_{3}^{Q}+\Lambda_{4}^{c}\right)^{2}+\left(h_{t}^{L}-\Lambda_{4}^{c}+\Lambda_{4}^{s}\right)^{2}\right)\left(\log \left(\frac{3 T}{m_{Q_{3}}}\right)+c+\frac{1}{2}\right)\right) \\
& +\frac{T^{2}}{\left(16 \pi^{2}\right)}\left(\frac{2}{3} g_{s}^{2} h_{t}^{2} \sin ^{2} \beta+\frac{3}{8} g_{w}^{2} h_{t}^{2} \sin ^{2} \beta+\frac{3}{8} g_{w}^{4}-\frac{1}{16} g_{w}^{4}\right),
\end{aligned}
$$

where

$$
\begin{aligned}
h_{t}^{f} & =h_{t}^{2} \sin ^{2} \beta T\left(1-\frac{3}{8} \frac{1}{\left(16 \pi^{2}\right)}\left[\left(12 h_{t}^{2} \sin ^{2} \beta-6 g_{w}^{2}-\frac{64}{3} g_{s}^{2}\right) L_{f}\right.\right. \\
& \left.\left.+g_{w}^{2}(2+28 \log 2)-96 \lambda \log 2+16 h_{t}^{2} \sin ^{2} \beta \log 2-\frac{64}{9} g_{s}^{2}(4 \log 2-3)\right]\right), \\
c & =\frac{1}{2}\left[\ln \frac{8 \pi}{9}+\frac{\zeta^{\prime}(2)}{\zeta(2)}-2 \gamma\right] \approx-0.348725 .
\end{aligned}
$$

The exact values of the parameters $\Lambda_{H_{3}}$ and $\Lambda_{U_{3}}$ in eqs. (35) and (36), depend on the particle content of the theory and on the input parameters. In fact, the dependence on $\tan \beta$ and $m_{t_{R}}$, for the range of values we are interested in, is weak. The dependence on $m_{Q}$ and $m_{D}$ is stronger. For $m_{Q}=m_{D}=300 \mathrm{GeV}$, the corresponding values of the parameters are in the range $\Lambda_{H_{3}}=$ $(1.6-1.8) T$, and $\Lambda_{U_{3}}=(6.6-6.9) T$.

\subsection{Zero-Temperature Renormalization}

In order to complete the matching of the $3 \mathrm{D}$ parameters to the $4 \mathrm{D}$ physical parameters, we must renormalize the zero-temperature theory. We will not go into the details of the renormalization, but refer the reader to the literature in which the pole masses for the relevant particles of our calculation have been obtained considering the full particle spectrum of the MSSM [31, 32]. We use the expressions given in ref. [31], keeping only the top Yukawa coupling, in the appropriate (large- $m_{A}$ ) limit. We have kept all $\bar{\mu}$-dependent contributions to order $g_{i}^{4}\left(h_{t}^{4}\right)$ but have neglected the constant contributions, which are not multiplied by $h_{t}$ or $g_{s}$. In this way all explicit dependence on $\bar{\mu}$ is cancelled at one-loop when we relate the $3 \mathrm{D}$ parameters to pole masses. 
Another zero-temperature constraint that we impose is the stability of the physical vacuum. In principle, a metastable region exists in which the colour-breaking minimum is lower than the physical one at zero temperature. If the time for the transition to this lower minimum is greater than the age of the Universe then this region of parameter space is also acceptable. However, we will not consider these issues in the present paper. The constraint for absolute stability can be obtained by studying the effective potential at zero temperature [10, 15]. This gives the constraint $-m_{U}^{2} \leq\left(m_{U}^{c}\right)^{2}$, where

$$
m_{U}^{c}=\left(\frac{m_{h}^{2} v^{2} g_{s}^{2}}{12}\right)^{1 / 4}
$$

\section{Results}

With the previous results we can now analyse the phase transition. In fig. 目 we show the critical temperatures for the transitions in the $\phi$ - and $\chi$-directions as a function of the right handed stop pole mass $m_{\tilde{t}_{R}}$, for $\tan \beta=3,5,12$. We find that, for $m_{Q} \sim 300 \mathrm{GeV}$, there still is a region in which a two-stage phase transition can occur. This region is to the left of the crossing points of the curves. With respect to the work of ref. [14] the structure of the phase diagram is preserved, although it is slightly shifted towards higher values of the right stop mass. Note that there is a considerable difference between our values of the critical temperatures and those in ref. [14]. Our analysis concludes that the structure of the phase diagram is robust to small additional corrections. This structure is maintained also for $m_{Q}=m_{D}=1 \mathrm{TeV}$. The total effect does not substantially increase or decrease the range of values of the right handed stop mass for which a two-stage phase transition can occur. However, the exact location of this small range in the value of $m_{\tilde{t}_{R}}$ depends on the value of the third-generation left handed squark doublet mass. In fig. 2 we give the values of $\frac{v}{T}$ for three different values of $\tan \beta$. As expected, the strength of the phase transition has a weak dependence on the values of the scales that have been fixed in our calculation, and only slight

\footnotetext{
${ }^{11}$ In this case the third-generation left handed squark doublet and right handed sbottom are decoupled from the thermal bath. The relations obtained from the dimensional-reduction procedure can be deduced from all of the formulae presented in the previous sections. All contributions from these fields are suppressed at finite temperature; however, a residual dependence on $m_{Q}$ as a consequence of a zero-temperature effect persists for the scalar Higgs self coupling $\lambda[14,30]$.
} 
differences are observed with respect to previous analyses.

Figure 3 shows lines of $\frac{v}{T}=1$ for three different cases in the $m_{h}-m_{\tilde{t}_{R}}$ plane. The phase transition is sufficiently strong for electroweak baryogenesis to the left of the solid (dotted) line for $m_{Q}=300$ $\mathrm{GeV}\left(m_{Q}=1 \mathrm{TeV}\right)$, using the results obtained in this paper. The dashed line is the result using the approximations of ref. [14], for $m_{Q}=300 \mathrm{GeV}$. We can see that the full effect of the corrections we have included on the strength of the phase transition is small. The end-points of the lines correspond to the maximum value of the Higgs mass that is reached by the effect of the zerotemperature radiative corrections for a given value of $m_{Q}$, and the $y$-axis starts at the value of the experimental limit on the Higgs mass

The allowed region in parameter space is shown in figs. 4 and 5, given the current experimental limits on the Higgs mass, for two different values of $m_{Q}$. The region on the left of the solid line indicates when a sufficiently strong first-order phase transition occurs. The dotted line gives the condition for absolute stability of the physical vacuum. As explained above, to the left of this line the colour-breaking minimum is lower than the physical one at zero-temperature. The dashed line is obtained when the critical temperatures of the transitions in the $\phi$ - and $\chi$-directions are the same. A two-stage phase transition occurs to the left of the dashed line. Note that, unlike the results of ref. [15] for $m_{Q}=1 \mathrm{TeV}$ and zero squark mixing, the dashed and solid lines do not intersect.

As mentioned in the introduction, for a sufficiently heavy right handed stop field, an effective theory with a single light scalar Higgs doublet field can be constructed. In the appendix we give the formulae that modify the equations presented in the previous sections. There are two ways of constructing this effective theory. We can either integrate out the right handed stop field simultaneously with the other heavy fields, or perform a third stage of reduction and integrate out the right stop field separately. The basic change in the equations of the appendix will be to replace the 3D coupling and masses by the 3D barred couplings and masses of section 2.2. It is clear, as mentioned above, that the results obtained with these effective potentials are unreliable for low values of the right handed stop mass, as perturbation theory is no longer under control. In terms of the 3D parameters we note in particular that the difference between $\lambda_{H_{3}}$ and $\bar{\lambda}_{H_{3}}$ is considerable, owing to the difference in $m_{U_{3}}^{2}$ and $\bar{m}_{U_{3}}^{2}$. However, comparing the results obtained with these three separate

\footnotetext{
${ }^{12} \mathrm{As}$ we are working in the large- $m_{A}$ limit, the Standard Model bound on the Higgs mass is used.
} 
approximations, we can obtain a more precise value of the right handed stop mass, for which the effective theory with a single light scalar field is a valid description. Recall that the one-loop estimate gave as a lower limit the value $m_{\tilde{t}_{R}} \gtrsim 177 \mathrm{GeV}$ [24, 26]. In fig. 6 we present the plot of the ratio of the vacuum expectation value of the Higgs doublet to the temperature as a function of the right handed stop mass obtained for the three approximations for the effective potential mentioned above. In this plot we have taken $\tan \beta=5$. The solid line is the result obtained when using the 2-loop effective potential derived in the main part of the paper. The dotted line corresponds to the results obtained after integrating out the right stop field simultaneously with the other heavy fields. The dashed line is the result obtained when a separate third stage of reduction is performed. We see that only for very large values of the right handed stop mass are the results basically the same. As we move towards lower values of $m_{t_{R}}$, the lines very quickly start to diverge. In particular, we see that for $m_{\tilde{t}_{R}}=177 \mathrm{GeV}\left(m_{U} \sim 50 \mathrm{GeV}\right)$ the results using the effective potentials for a single light scalar are completely unreliable. To understand why the results of the dashed and dotted lines are so different for smaller values of $m_{\tilde{t}_{R}}$, we recall that the strength of the transition is dominated by the value of $\lambda_{H_{3}}$ in the final 3D effective theory for a single light scalar field at the phase transition. The value of $\lambda_{H_{3}}$ is much smaller when a third stage of reduction is performed, as the value of $m_{U_{3}}^{2}$ is much smaller too. That is, the net effect artificially strengthens the first-order phase transition.

\section{Conclusions}

We have performed a full two-loop dimensional reduction of 4D MSSM parameters to the 3D couplings and masses of the effective theory. In this way, we have fixed the scales appearing in the 3D mass terms that are due to the thermal polarizations and the super-renormalizability of the 3D theory. The values of the parameters $\Lambda_{H_{3}}$ and $\Lambda_{U_{3}}$ can vary significantly for different values of the input parameters and the particle content of the theory, thus modifying the critical temperatures of the transitions. We have compared our results with previous analyses. We conclude that the corrections relevant to the preservation of the baryon asymmetry are small and that the allowed

range of masses is $m_{h} \lesssim 110 \mathrm{GeV}$ and $m_{\tilde{t}_{R}} \lesssim m_{t}$, in complete agreement with previous results. We find that the phase structure diagram still allows a possible two-stage phase transition for a small range of values of $m_{\tilde{t}_{R}}$. This range of values is shifted compared to previous results. However, 
whether or not the transition actually occurs must be explicitly checked. Initial lattice analysis suggests that the second stage of the transition is extremely strong and thus this transition might not have taken place on cosmological time scales. Consequently, this region of parameter space for electroweak baryogenesis would be excluded. From the comparison of the results obtained using the different approximations to the effective potential we can conclude that for $\tilde{m}_{t_{R}} \lesssim 210 \mathrm{GeV}$, the results obtained for the strength of the phase transition with a single light Higgs field at the transition point are unreliable.

\section{Acknowledgements}

I would like to thank M. Shaposhnikov, C. Wagner and in particular M. Laine for many useful discussions. I especially thank J.R. Espinosa for interesting discussions and comments on the manuscript.

\section{Appendix}

\section{Integrating out the right handed stop}

The additional corrections that must be included in the case of integrating out the right handed stop are given below. The Higgs self-coupling is modified by

$$
\bar{\lambda}_{3}=\lambda_{3}-\frac{3}{16 \pi} \gamma_{3}^{2} \frac{1}{m_{U_{3}}} .
$$

The one-loop correction to the mass term is

$$
\bar{m}_{3}^{2}=m_{3}^{2}-\frac{3}{4 \pi} \gamma_{3} m_{U_{3}} .
$$

The relevant contributions from the 2-loop graphs have been given in ref. [12 with the following modifications, which include the effects of the dimensional-reduction procedure. For the (SSV) and $(\mathrm{SV})$ terms substitute $g_{s} \rightarrow g_{s_{3}}$, for the (SSS) term substitute $h_{t}^{2} \sin ^{2} \beta \rightarrow \gamma_{3}^{2}$ for the (SS) term substitute $\frac{g_{s}^{2}}{6} \rightarrow \lambda_{U_{3}}$ and $h_{t}^{2} \sin ^{2} \beta \rightarrow \gamma_{3}^{2}$. 


\section{References}

[1] V.A. Kuzmin, V.A. Rubakov, and M.E. Shaposhnikov. Phys. Lett., B155:36, 1985.

[2] M.E. Shaposhnikov. Nucl. Phys., B287:757, 1987.

[3] A. Cohen, D.B. Kaplan, and A.E. Nelson. Annu. Rev. Nucl. Part. Sci., 43:27, 1993.

[4] V.A. Rubakov and M. Shaposhnikov. Usp. Fiz. Nauk, 166:493, 1996.

[5] M. Quiros. Helv. Phys. Acta, 67:451, 1994.

[6] S. Myint. Phys. Lett., B287:325, 1992.

[7] G.F. Giudice. Phys. Rev., D45, 1992.

[8] J.R. Espinosa, M. Quirós, and F. Zwirner. Phys. Lett., B307:106, 1993.

[9] A. Brignole, J.R. Espinosa, M. Quirós, and F. Zwirner. Phys. Lett., B324:181, 1994.

[10] M. Carena, M. Quiros, and C.E.M. Wagner. Phys. Lett., B380:81, 1996.

[11] D. Delepine, J.-M. Gerard, R. Gonzalez Felipe, and J. Weyers. Phys. Lett., B386:183, 1996.

[12] J.R. Espinosa. Nucl. Phys., B475:273, 1996.

[13] B. de Carlos and J.R. Espinosa. Nucl. Phys., B503:24, 1997.

[14] D. Bodeker, P. John, M. Laine, and M.G. Schmidt. Nucl. Phys., B497:387, 1997.

[15] M. Carena, M. Quiros, and C.E.M. Wagner. preprint (hep-ph/9710401), 1997.

[16] J. Cline and G.D. Moore. preprint (hep-ph/9806354), 1998.

[17] P. Ginsparg. Nucl. Phys., B170:388, 1980.

[18] T. Applequist and R. Pisarski. Phys. Rev., D23:2305, 1981. 
[19] S. Nadkarni. Phys. Rev., D27:917, 1983.

[20] K. Farakos, K. Kajantie, K. Rummukainen, and M.E. Shaposhnikov. Nucl. Phys., B425:67, 1994.

[21] K. Kajantie, M. Laine, K. Rummukainen, and M.E. Shaposhnikov. Nucl. Phys., B458:90, 1996.

[22] A. Jakovác and A. Patkós. Phys. Lett., B334:334, 1994.

[23] E. Braaten and A. Nieto. Phys. Rev., D51:6990, 1995.

[24] M. Laine. Nucl. Phys., B481:43, 1996.

[25] J. Cline and K. Kainulainen. Nucl. Phys., B482:73, 1996.

[26] M. Losada. Phys. Rev., 56:2893, 1997.

[27] G. Farrar and M. Losada. Phys. Lett., B406:60, 1997.

[28] M. Losada. in preparation.

[29] P. Arnold and O. Espinosa. Phys. Rev., D47:3546, 1993.

[30] M. Laine and K. Rummukainen. preprint (hep-ph/9804255), (hep-lat/9804019), 1998.

[31] D. Pierce, J. Bagger, K. Matchev, and R. Zhang. Nucl. Phys., B491:3, 1997.

[32] A. Donini. Nucl. Phys., B467:3, 1996.

[33] V. Ruhlmann-Kleider, DELPHI Collaboration. Contribution to the Moriond Conference on Electroweak Interactions, 1998.

http://delphiwww.cern.ch/delfigs/searches/vanina980325/mor98.ps 


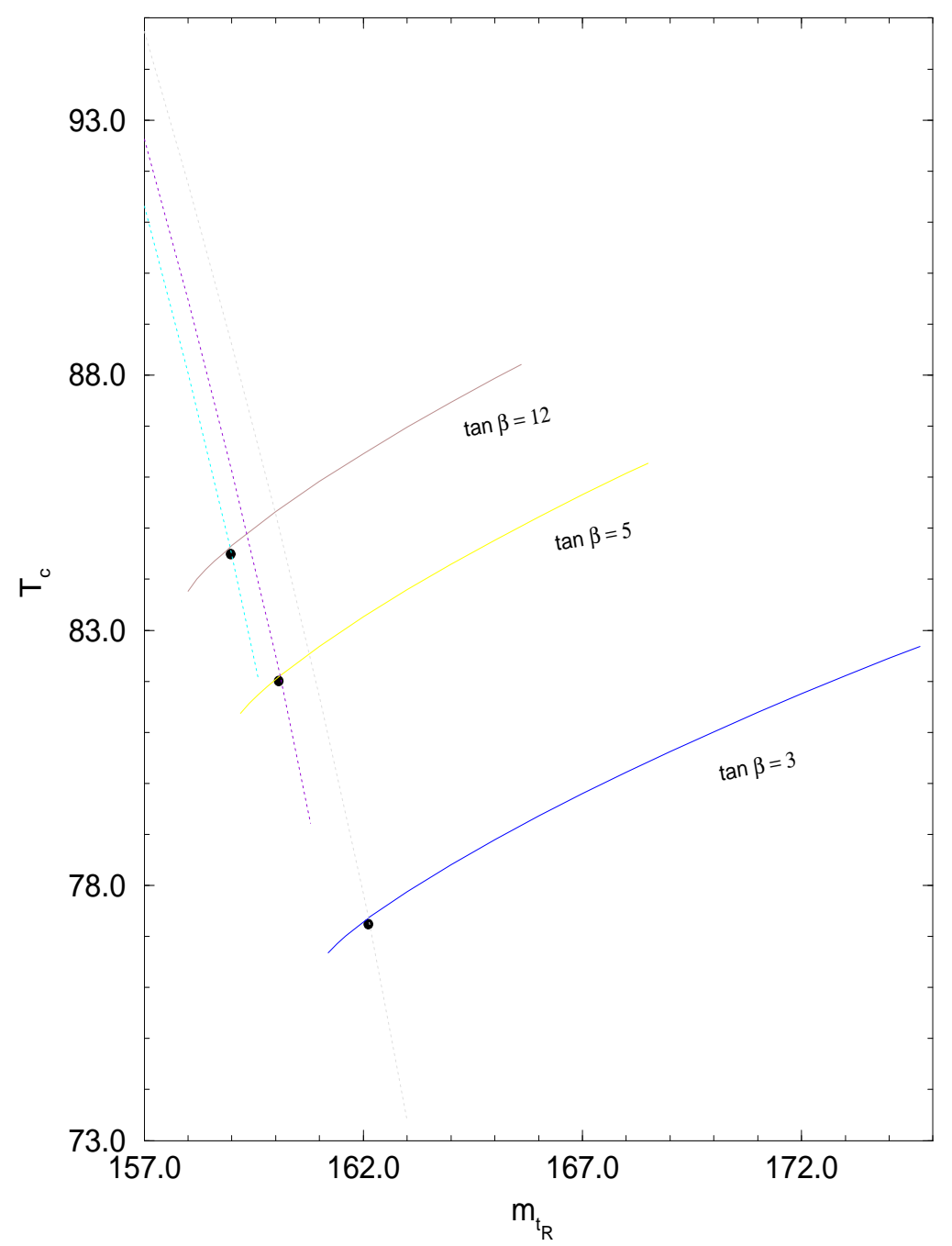

Figure 1: Critical temperatures in the $\phi$ - (solid) and $\chi$ - (dotted) directions as functions of $m_{\tilde{t}_{R}}$ for $\tan \beta=3,5,12$ and $m_{Q}=300 \mathrm{GeV}$. 


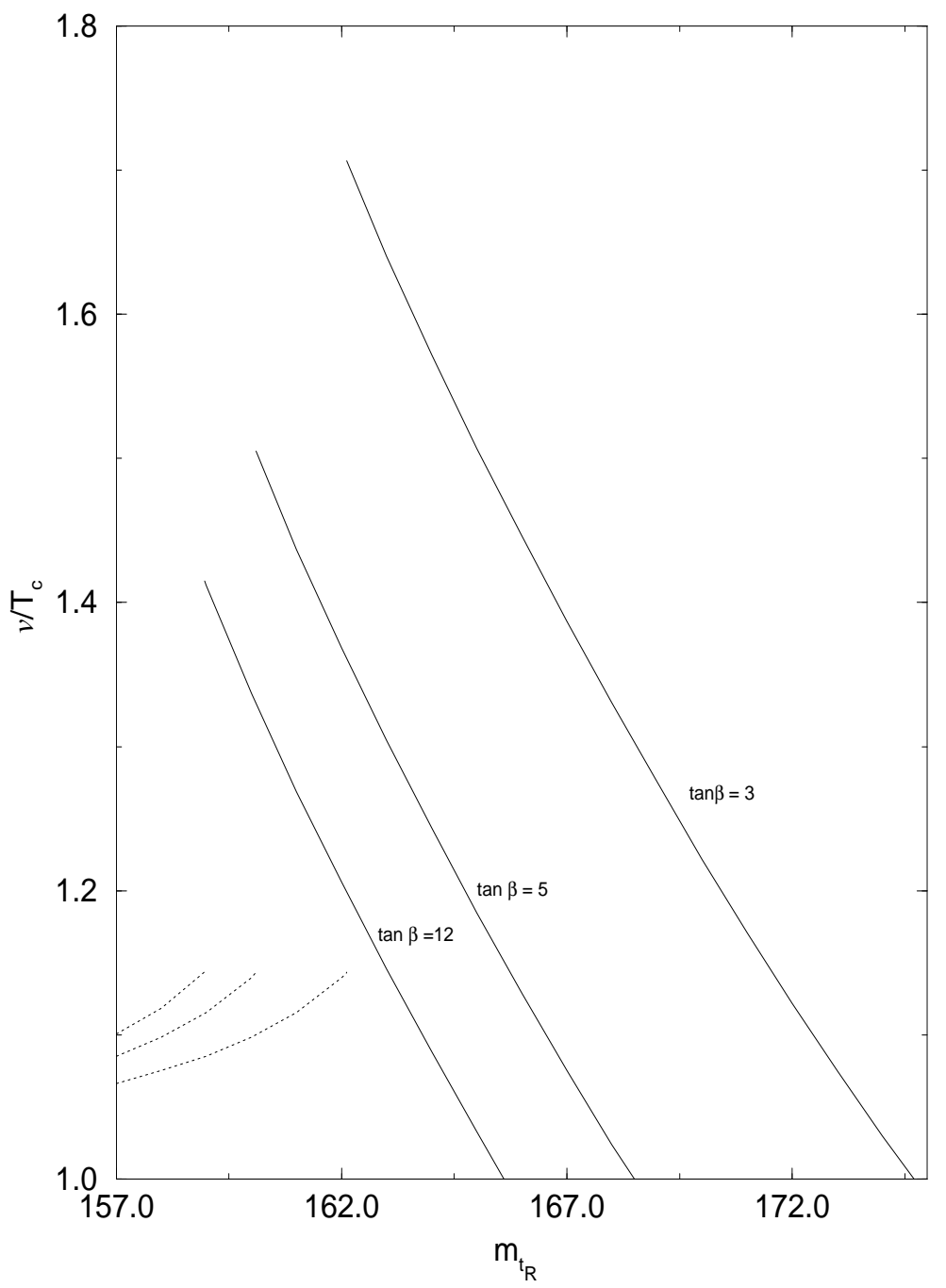

Figure 2: Plot of $\frac{v}{T}$ as a function of $m_{\tilde{t}_{R}}$ in the $\phi$ - (solid line) and $\chi$ - (dotted line) directions for $\tan \beta=3,5,12$ and $m_{Q}=300 \mathrm{GeV}$. For a given value of $\tan \beta$ the lines end at the same value of the right handed stop mass. 


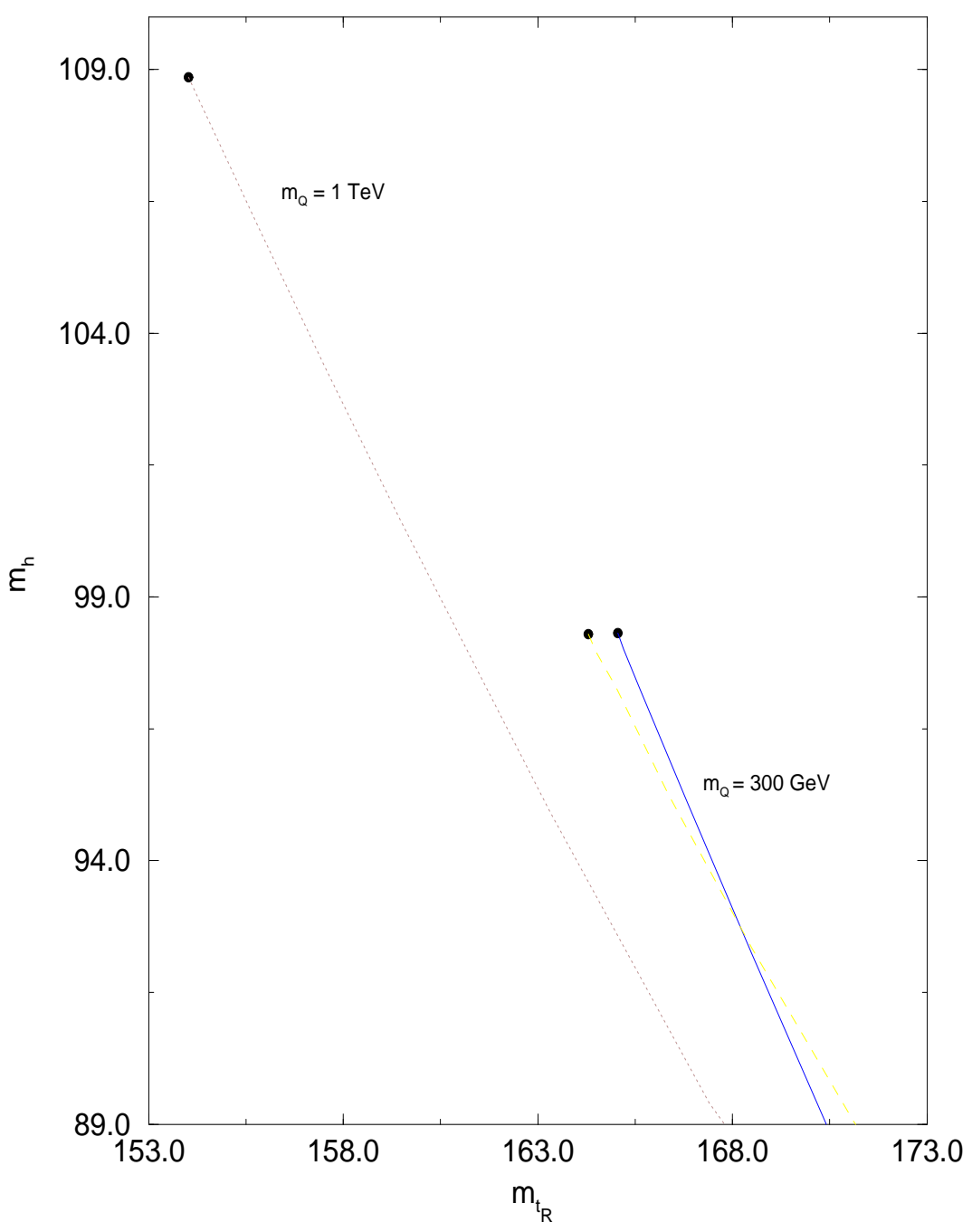

Figure 3: Contours of $\frac{v}{T}=1$ in the $m_{h}-m_{\tilde{t}_{R}}$ plane. The solid (dotted) line corresponds to the results obtained within our approximations for $m_{Q}=300 \mathrm{GeV}(1 \mathrm{TeV})$. The dashed line is the result using the approximations of ref. [14] for $m_{Q}=300 \mathrm{GeV}$. The region to the left of the lines gives a sufficiently strong first-order phase transition, for a given value of $m_{Q}$. 


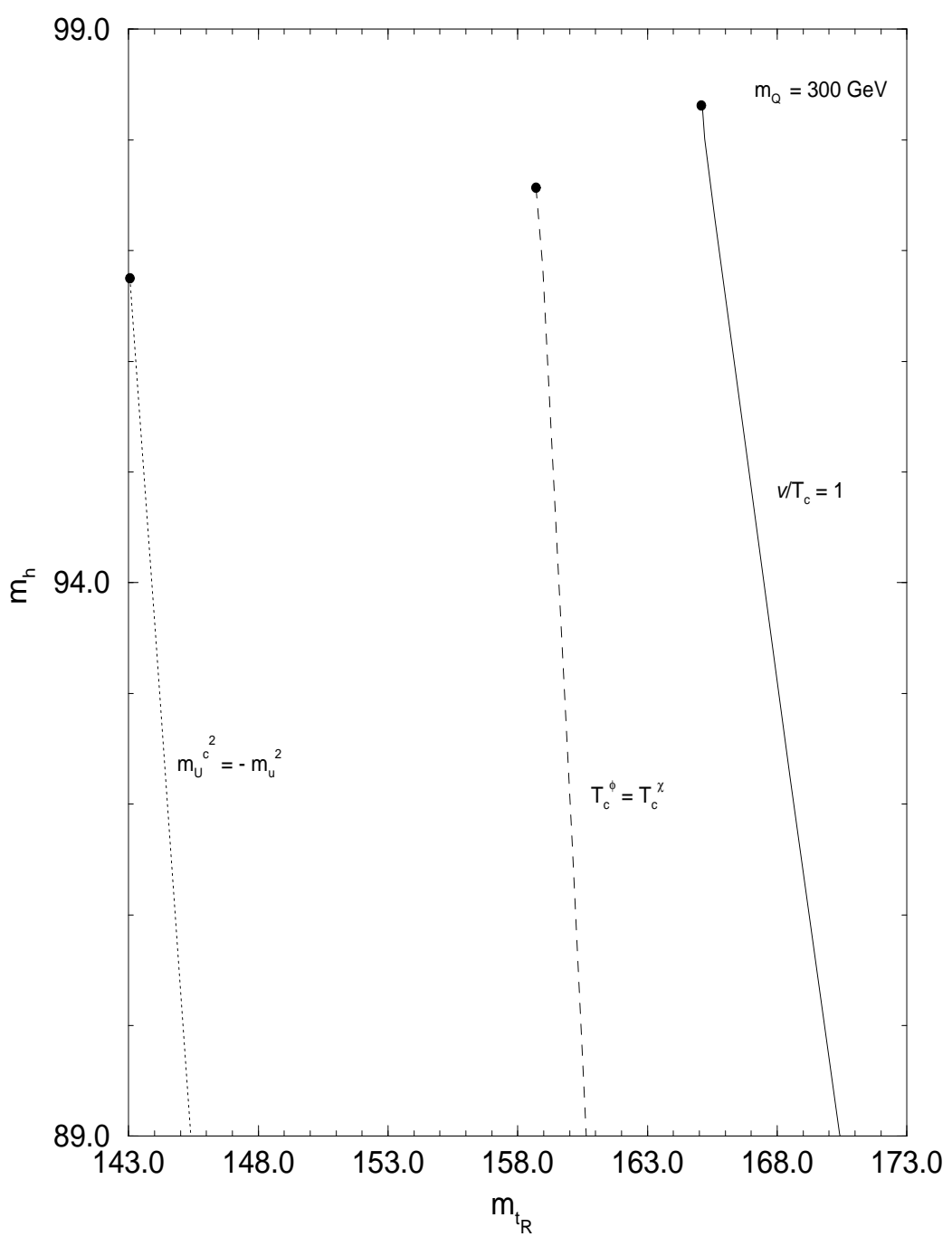

Figure 4: Allowed region in $m_{h}-m_{\tilde{t}_{R}}$ plane for $m_{Q}=300 \mathrm{GeV}$. To the left of the solid line there is a sufficiently strong first-order phase transition, to the right of the dotted line the physical vacuum is absolutely stable. The dashed line separates the region for which a two-stage phase transition can occur. 


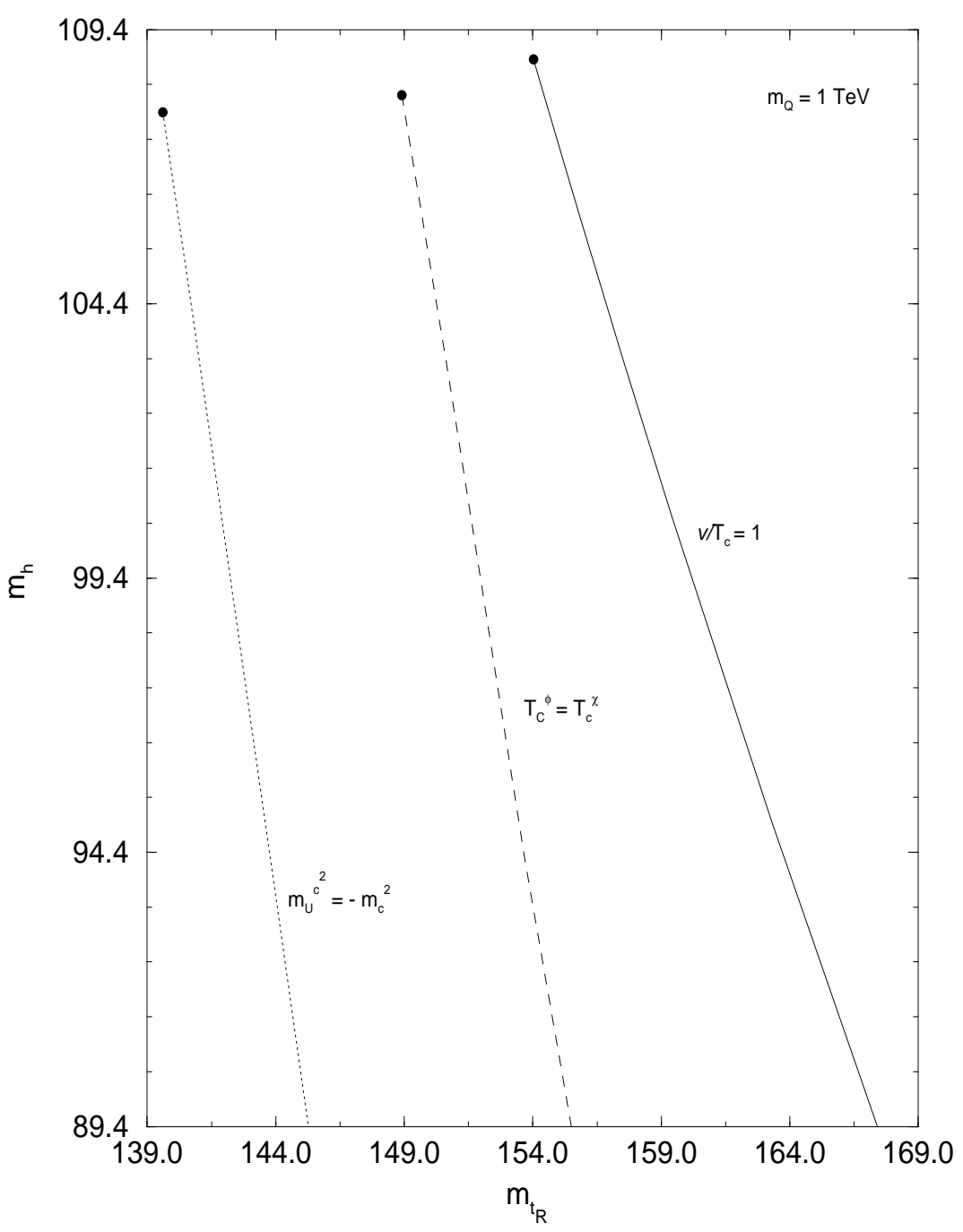

Figure 5: Same as fig. 因, for $m_{Q}=1 \mathrm{TeV}$. 


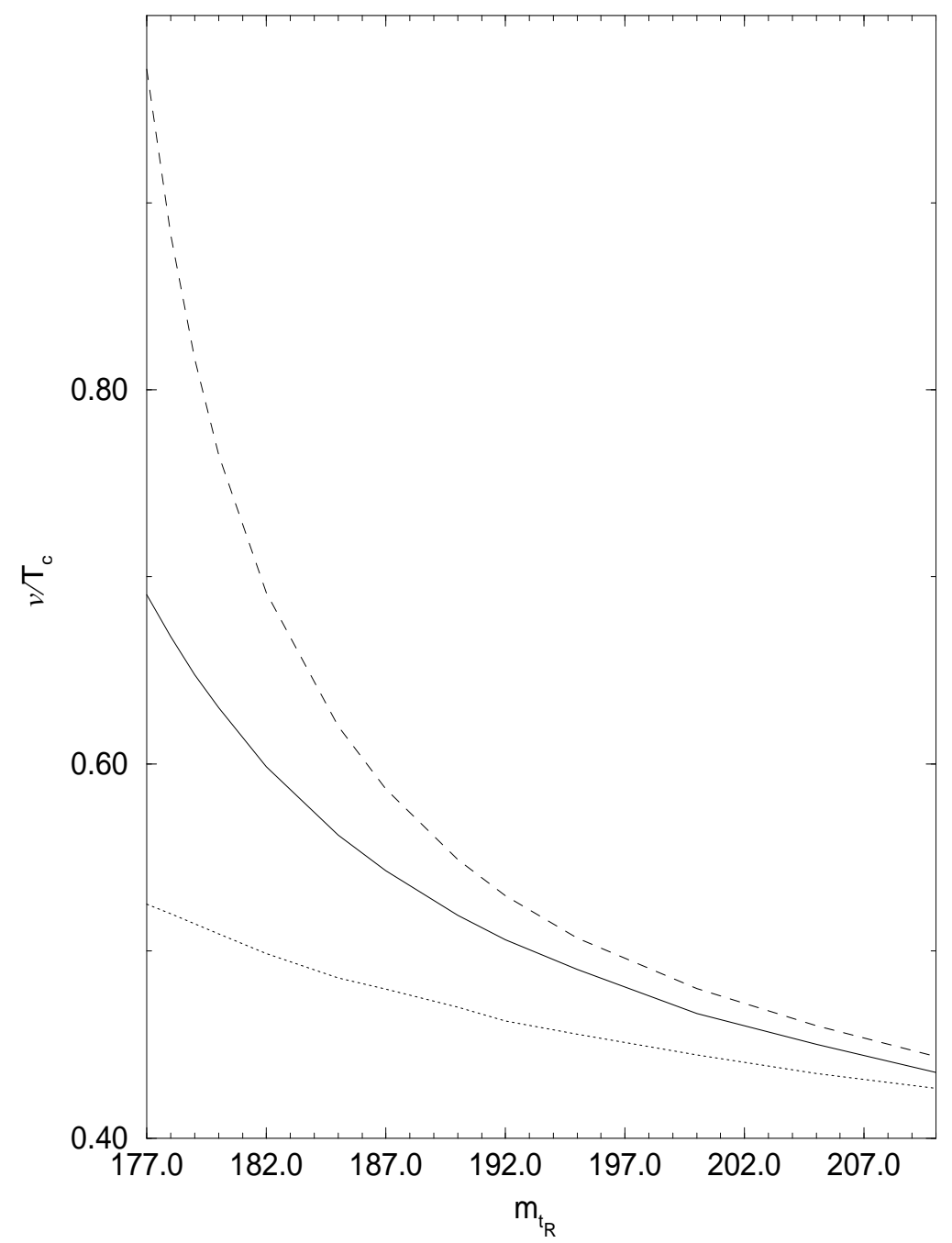

Figure 6: Plot of $\frac{v}{T}$ as a function of $m_{\tilde{t}_{R}}$ in the $\phi$-direction for three cases. The solid line corresponds to the result obtained with the effective potential given in the main part of the paper. The dotted line corresponds to the results obtained after integrating out the right stop field simultaneously with the other heavy fields. The dashed line is the result obtained when a separate third stage of reduction is performed. 